\title{
Structural basis of GIRK2 channel modulation by cholesterol and $\mathrm{PIP}_{2}$
}

Yamuna Kalyani Mathiharan ${ }^{2, \star}$, lan W. Glaaser ${ }^{1, \star}$, Yulin Zhao ${ }^{1}$, Michael J. Robertson ${ }^{2}$, Georgios Skiniotis $^{2, \#}$, Paul A. Slesinger,"\#.

${ }^{1}$ Nash Family Department of Neuroscience, Icahn School of Medicine at Mount Sinai, New York, NY, USA,

${ }^{2}$ Department of Molecular and Cellular Physiology; Department of Structural Biology, Stanford University School of Medicine, Stanford, CA, USA,

${ }^{\star}$ These authors contributed equally to this work

\#Address correspondence to: Georgios Skiniotis (yiorgo@stanford.edu), Paul A. Slesinger (paul.slesinger@mssm.edu)

\section{Significance/Impact:}

- GIRK2 is one of the primary GIRK channels in brain. Characterization of cholesterol binding in GIRK2 provides new therapeutic sites for modulating these channels.

- Many ion channels are modulated by cholesterol, but the molecular basis of this modulation has been elusive.

- First cryoEM structures of pore-forming Kir alpha subunit.

\section{Keywords:}

CryoEM, GIRK, inwardly rectifying potassium channel, cholesterol, lipids, $\mathrm{PIP}_{2}$

Word counts: Abstract (136) - Main text (3048) 


\section{ABSTRACT}

G protein-gated inwardly rectifying potassium (GIRK) channels play important roles in controlling cellular excitability in the heart and brain. While structural data begin to unravel the molecular basis for $\mathrm{G}$ protein and alcohol dependent activation of GIRK channels, little is known about the modulation by cholesterol. Here, we present cryo-electron microscopy (cryoEM) structures of GIRK2 in the presence and absence of the cholesterol analog cholesteryl hemisuccinate $(\mathrm{CHS})$, and $\mathrm{PIP}_{2}$. The structures and their comparison reveal that $\mathrm{CHS}$ binds near $\mathrm{PIP}_{2}$ in lipid-facing hydrophobic pockets of the transmembrane domain (TMD). CHS potentiates the effects of $\mathrm{PIP}_{2}$, which stabilizes the inter-domain region and promotes the engagement of the cytoplasmic domain (CTD) onto the transmembrane region. The results suggest that $\mathrm{CHS}$ acts as a positive allosteric modulator and identify novel therapeutic sites for modulating GIRK channels in the brain. 
G protein-gated inwardly rectifying potassium (GIRK) channels provide a major source of inhibition in the brain, and have been implicated in a variety of neurological disorders ${ }^{1}$. Loss of GIRK channels leads to hyperexcitability and susceptibility to seizures, changes in alcohol consumption, and increases in sensitivity to psychostimulants ${ }^{2-6}$. Activation of $\mathrm{G}$ protein-coupled receptors (GPCRs) that signal through $\mathrm{G \alpha}_{\mathrm{i} / \mathrm{o}} \mathrm{G}$ protein, such as $\mathrm{GABA}_{\mathrm{B}}$, D2 dopamine and $\mathrm{m} 2$ muscarinic receptors, leads to liberation of $G$ protein $G \beta \gamma$ subunits that open GIRK channels through direct protein-protein interactions ${ }^{7-9}$. GIRK channels are also activated directly by alcohol $^{10-12}$. Recently, GIRK channels have been shown to be modulated by membrane cholesterol $^{13-15}$, similar to other ion channels such as calcium-activated potassium channels ('BK") and TRP channels ${ }^{15,16}$. GIRK currents are significantly larger in Xenopus oocytes enriched with cholesterol ${ }^{14,15}$ and, in a minimal lipid vesicle reconstitution experiment with only GIRK2 and $\mathrm{PIP}_{2}$, the addition of cholesterol significantly enhances potassium conductance ${ }^{13}$.

Some of the questions on the molecular aspects of GIRK channel gating have been addressed through a combination of electrophysiology, biochemistry, mutagenesis and determination of high-resolution structures. For example, G $\beta \gamma$ binds directly to the channel via the $\beta L-\beta M$ loop in the cytoplasmic domain (CTD $)^{17-20}$. The binding induces a conformational change in the channel's two gates, the G loop gate ${ }^{21}$ and the helix bundle crossing $(\mathrm{HBC})$ gate $^{22}$, widening the pore to enable $\mathrm{K}^{+}$permeation, as suggested by the X-ray crystal structures of GIRK2 in complex

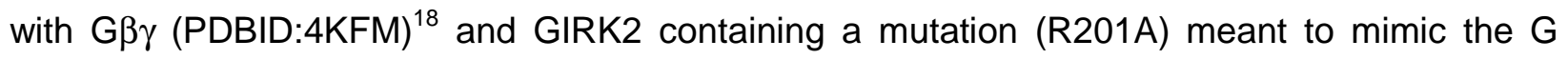
protein activated state (PDBID:3SYQ $)^{23}$. A physical binding pocket for mediating alcoholdependent activation has been also localized in the CTD, formed by the three domains at the subunit interface, the $\beta \mathrm{L}-\beta \mathrm{M}$ loop from one subunit and the $\beta \mathrm{D}-\beta \mathrm{E}$ loop and part of the $\mathrm{N}$ terminal domain from the other subunit ${ }^{11,24}$. The activation of GIRK channels by GßY and alcohol both require the membrane phospholipid $\mathrm{PIP}_{2}$, which interacts with a cluster of basic residues located at the inner membrane leaflet near the cytoplasmic side of the M1 and M2 
transmembrane helices ${ }^{23,25}$. The mechanism by which $\mathrm{PIP}_{2}$ supports channel activation, however, is poorly understood, partly due to structural differences observed in the apo state (i.e., no $\mathrm{PIP}_{2}$ ) of inward rectifier channels ${ }^{23,26}$.

Moreover, the mechanism of modulation of GIRK channels by cholesterol is unclear as no binding sites have been directly determined by structural studies. Cholesterol was originally hypothesized to alter the function of proteins in the plasma membrane through changes in membrane stiffness ${ }^{27,28}$. However, more recent studies suggest that cholesterol exerts specific effects through direct protein engagement ${ }^{27,28}$. Putative cholesterol-binding motifs (e.g., CRAC, CARC, CCM) have been identified in some proteins (for review, see ${ }^{27}$ ). Consistent with this, several structures of GPCRs have shown cholesterol bound to a hydrophobic region of the receptor $^{27,29-33}$. Mutagenesis studies and simulations have implicated several different regions of inwardly rectifying potassium channels in cholesterol modulation, including the cytoplasmic domain, $\mathrm{PIP}_{2}$ binding site, and the transmembrane domain ${ }^{14,34-37}$. However, the exact location of the cholesterol sites remains to be determined.

To better understand the structural basis for cholesterol and $\mathrm{PIP}_{2}$ modulation of GIRK channels, we employed cryo-electron microscopy (cryoEM) to visualize GIRK2 under different conditions. Here, we present structures of the GIRK2 channel in the presence and absence of the cholesterol derivative cholesteryl hemisuccinate $(\mathrm{CHS})$ and $\mathrm{PIP}_{2}$, revealing their effects on mechanistic aspects of GIRK function.

\section{RESULTS}

CHS potentiates GIRK2 channel activity

Previously, we showed that GIRK2 channels are potentiated by cholesterol in the absence of G proteins $^{13}$. To better understand the mechanism of cholesterol modulation, we sought to 
determine the site of interaction of a cholesterol analog, cholesteryl hemisuccinate (CHS), in GIRK2 channels. To verify the functional effects of CHS, we employed Mus musculus GIRK2 recombinantly expressed in Pichia pastoris and extracted from membranes with n-Dodecyl $\beta$-Dmaltoside (DDM) detergent in the presence or absence of CHS (Extended Fig. S1a). Purified GIRK2 tetrameric channels were reconstituted into liposomes containing $1 \%$ brain $\mathrm{PIP}_{2}$ alone, which is required for channel function ${ }^{13,38}$, and either $2.5 \% \mathrm{CHS}$ or $5 \%$ cholesterol. We measured $\mathrm{K}^{+}$permeation through GIRK2 channels using a flux assay (Fig. 1a), as described previously ${ }^{13,18}$. Upon addition of CCCP, the fluorescence emitted from ACMA pre-loaded into proteoliposomes containing GIRK2 channels and 1\% brain $\mathrm{PIP}_{2}\left(\mathrm{GIRK} 2^{\mathrm{PIP} 2}\right)$ quenches to 0.5 F/Fo under basal conditions (Fig.1a and b). Next, we reconstituted membranes in CHS $\left(\mathrm{GIRK}^{\mathrm{PIP} 2 / \mathrm{CHS}}\right)$ and observed fluorescence quenching increasing to $\sim 0.2 \mathrm{~F} / \mathrm{F}_{0}$ following $\mathrm{CCCP}$, indicating potentiation of GIRK2 channel activity (Fig.1a and b). The GIRK2 channel-specific inhibitor, MTS- $\mathrm{HE}^{13}$, reduced the extent of quenching, indicating inhibition of the $\mathrm{K}^{+}$ conductance. We used the inhibition with MTS-HE to quantify changes in quenching under different conditions and converted this to a percentage of relative $\mathrm{K}^{+}$flux. Under basal conditions with proteoliposomes containing GIRK2 channels and $1 \%$ brain $\mathrm{PIP}_{2}\left(\mathrm{GIRK} 2^{\mathrm{PIP} 2}\right)$, the $\%$ relative $\mathrm{K}^{+}$flux was $44.0 \% \pm 2.3 \%(\mathrm{n}=18)$ (Fig. 1C-e). Both CHS and cholesterol significantly increased the relative $K^{+}$flux to $65.2 \% \pm 0.4 \%(n=10)$ and $69.4 \% \pm 0.3 \%(n=17)$, respectively, compared to basal conditions (Figure 1c-e). Taken together, these results demonstrate that $\mathrm{CHS}$, like cholesterol, potentiates basal $\mathrm{PIP}_{2}$-dependent GIRK2 channel activity ${ }^{13}$.

\section{Structure of GIRK2 in the presence of CHS and $\mathrm{PIP}_{2}$}

To gain insights to CHS binding in GIRK2, we proceeded with optimizing the sample for cryoEM studies. The sample quality was assessed by size-exclusion chromatography (Extended Fig. S1a) and further evaluated by negative stain electron-microscopy (Extended Fig. S1b) to identify conditions with predominantly well-formed GIRK2 tetramers ${ }^{39}$. To ensure the presence 
of $\mathrm{CHS}$ and $\mathrm{PIP}_{2}\left(\mathrm{GIRK} 2^{\mathrm{PIP} 2 / \mathrm{CHS}}\right)$, the sample was purified in the presence of $\mathrm{CHS}$ and incubated with $2 \mathrm{mM}$ of $\mathrm{diC}_{8}-\mathrm{PIP}_{2}$ prior to cryoEM grid preparation.

We subsequently employed cryoEM and determined the structure of GIRK2 $2^{\mathrm{PIP} / \mathrm{CHS}}$ at a global indicated resolution of $3.5 \AA$ (Extended Fig. S2). The structure revealed an overall architecture similar to those solved by X-ray crystallography ${ }^{18,23}$, with two distinct domains, the transmembrane (TMD) and cytoplasmic (CTD), and the pore along the four-fold axis with wellordered inner-helix bundle crossing and G-loop gates (Fig. $\mathbf{2 a}$ and $\mathbf{2 b}$ ). $\mathrm{PIP}_{2}$ is bound at its canonical site near inter-domain linkers, as also observed in other inwardly rectifying potassium channels $^{18,23,26}$ (Fig. 2b). Interestingly, we observed a planar density on the opposite side of the $\mathrm{PIP}_{2}$-channel interface that is consistent with $\mathrm{CHS}$, hereafter referred to as $\mathrm{CHS} 1$. Initial modeling of $\mathrm{CHS}$ in the EM density was performed with the GemSpot pipeline ${ }^{40}$, followed by manual refinement in COOT $^{41}$. This approach yielded largely identical poses for $\mathrm{CHS}$ in good agreement to the EM map and chemically plausible interactions with GIRK2 transmembrane helices that had subtle differences in the orientation of iso-octyl tail (Fig. $\mathbf{2 b}$-d and Extended Fig. S3a). The head group of CHS1 is in position to form a salt bridge with R92, while its sterane rings and iso-octyl tail are stacked against the TMD, surrounded by hydrophobic residues F93, L95, L96, V99 of M1 helix from one subunit, and I175, V178, L179 of M2 helix from the adjacent subunit (Fig. 2b-d and Extended Fig. S3b). Another CHS molecule (CHS2) could be modelled near the N-terminus surrounded by hydrophobic residues that include V72, L79, I82, L86, L89, 197, V101 and F186 (Fig. 2b-d and Extended Fig. S3c). We cannot rule out other putative CHS sites, as additional densities are observed near the TMD-CTD interface, but those are poorly resolved (Extended Fig. S3d).

Structures of GIRK2 $2^{P I P 2}$ in the absence of $\mathrm{CHS}$ 
To assess whether CHS alters the interaction of $\mathrm{PIP}_{2}$, we next determined the structure of GIRK2 $^{\text {PIP2 }}$ in the absence of CHS during the purification. Interestingly, cryoEM analysis resolved different conformers of GIRK2 ${ }^{\mathrm{PIP} 2}$ (Extended Fig. S4). The predominant conformer, referred to as GIRK2 ${ }^{\text {PIP2* }}$, accounted for $~ 39 \%$ of well-defined particles and its structure was obtained at a global indicated resolution of $3.2 \AA$. This structure is comparable to GIRK2 ${ }^{\mathrm{PIP} 2 / \mathrm{CHS}}$ (Fig. 3), with four $\mathrm{PIP}_{2}$ molecules bound at equivalent sites, but lacked densities for $\mathrm{CHS} 1$ and $\mathrm{CHS} 2$ (Extended Fig. 3d). A second conformer, GIRK2 ${ }^{\mathrm{PIP} 2 * *}$, accounting for $\sim 27 \%$ of projections was determined at $4.8 \AA$ global resolution. Comparison of this conformation with GIRK2 ${ }^{\mathrm{PIP} 2 *}$ reveal that the CTD is twisted from $8-12^{\circ}$ around the four-fold axis and is partially disengaged from the TMD by 1-2 $\AA$ (as evaluated by the distance between Ca atoms of I195 and Q322), with a stretched tether helix (residues 197-203, Extended Fig. S5a-c).

The disengagement of the CTD is more pronounced in two additional conformers, GIRK2 $2^{\text {PIP2*** }}$ and GIRK2 $2^{\mathrm{PIP} 2 * \star \star *}$, as the distance between $\mathrm{Ca}$ atoms of $\mathrm{I195}$ and Q322 is increased by 3-8 $\AA$ (Extended Fig. S5a and b). These conformers appear very similar and were obtained at low overall resolution $(\sim 7.7 \AA)$, primarily due to the relative flexibility between TMDs and CTDs, but nevertheless rigid body docking was sufficient in revealing the overall backbone configurations. Interestingly, though the CTD detachment is different in each subunit, its C4 symmetry appears nearly preserved in all the conformers. We next examined whether the interaction of $\mathrm{PIP}_{2}$ in its binding pocket was altered by the presence of $\mathrm{CHS}$ (Fig. 3a). The $1^{\prime} \mathrm{PO}_{4}$ of the $\mathrm{PIP}_{2}$ anionic headgroup is coordinated by the side chain of positively charged $\mathrm{R} 92$ while the $5^{\prime} \mathrm{PO}_{4}$ with K194, K199, K200, and O6 atom with the backbone amide group of W91 (Fig. 3ai). Positively charged residues $\mathrm{K} 64$ and $\mathrm{K} 90$ are also in proximal distance to the anionic headgroup of $\mathrm{PIP}_{2}$. This configuration of $\mathrm{PIP}_{2}$ looks similar to that in GIRK2 $2^{\mathrm{PIP} 2 / \mathrm{CHS}}$ (Fig. 3aii), but has lower occupancy. Taken together, these observations suggest that the inter-domain interface of GIRK2 $^{\mathrm{PIP} 2}$ is relatively less stable than that of GIRK2 $2^{\mathrm{PIP} 2 / \mathrm{CHS}}$ (Extended Fig. S5a and b). 
In both the GIRK2 $2^{\mathrm{PIP} 2 \star}$ and GIRK2 $2^{\mathrm{PIP} 2 / \mathrm{CHS}}$ maps, we observe densities that are compatible with $\mathrm{Na}^{+}$and $\mathrm{K}^{+}$ions. $\mathrm{A} \mathrm{Na}{ }^{+}$ion is coordinated by $\mathrm{D} 228$, as shown previously in X-ray crystal structures ${ }^{23}$. Similarly, densities for $\mathrm{K}^{+}$ions are found near the selectivity filter, Y266, M319, G318 and E236 as previously determined ${ }^{18,23}$ but also observed at new positions near G158, F192 and M313 (Fig. 3b). We also note the presence of additional, less well resolved densities along the pore near N184, V188, E236 and M319. Thus, the channel with four $\mathrm{PIP}_{2}$ molecules bound at the TMD-CTD appears to be in a state favorable for conducting $\mathrm{K}^{+}$ions through the pore.

Apo GIRK2 structure (no $\mathrm{PIP}_{2}$ )

Lastly, we examined the structure of apo GIRK2, in the absence of modulators $\mathrm{PIP}_{2}$ and $\mathrm{CHS}$. CryoEM maps of apo GIRK2 determined at overall $4.8 \AA$ resolution revealed that the CTD is detached from the TMD (Fig. 4 and 5). This configuration of the CTD is quite different from the apo GIRK2 structure determined by X-ray crystallography, which showed the CTD engaged with the $\mathrm{TMD}^{23}$ (Extended Fig. S5d). Inter-domain linkers consisting of tether helix and N-terminal residues 67-78 appear mostly disordered, suggesting that this interface is unstable in the absence of $\mathrm{PIP}_{2}$ and CHS (Fig.5; Extended Fig. S5e, S6 and Extended Movie S1). The gap between the CTD and TMD is 23-28 $\AA$ (Fig. 4). The detached CTD appears tilted below the membrane, reflecting 'wobble' in this structure and an overall asymmetry in the absence of $\mathrm{PIP}_{2}$ and CHS. Thus, our cryoEM studies reveals a new apo GIRK2 closed state.

\section{DISCUSSION}

The potential connection between elevated plasma cholesterol and increased risk for cardiac disease is well known ${ }^{42}$. Less well understood is the role of cholesterol in the brain. Nearly all cholesterol in the CNS is derived from de novo synthesis, and is therefore not influenced by 
dietary changes in cholesterol $^{43}$. Nevertheless, changes in brain cholesterol have been implicated in numerous neurodegenerative diseases, such as Alzheimer's disease (AD), Huntington's disease, Parkinson's disease, and Niemann-Pick disease ${ }^{44}$. For example, hypercholesterolemia causes impairment of dopamine signaling and psychomotor dysfunction in mice ${ }^{45-48}$. Increases in cholesterol levels have been shown to elevate beta-amyloid precursor protein levels in cholesterol-enriched lipid rafts $^{49}$ and are associated with increased risk for $A D^{50}$. Accordingly, there is a growing need to understand how cholesterol affects brain function.

\section{Cholesterol binding sites on membrane proteins}

Cholesterol binding sites on membrane proteins were initially characterized using a variety of techniques, including electron spin resonance, photoaffinity labeling, molecular modeling and site-directed mutagenesis, which led to the identification of amino acid recognition motifs ${ }^{15,37,51 \text { - }}$ 54. CRAC (Cholesterol Recognition/interaction Amino acid Consensus) is characterized by the amino acid sequence $(\mathrm{L} / \mathrm{V})-\mathrm{X}_{1-5^{-}}(\mathrm{Y})-\mathrm{X}_{1-5^{-}}(\mathrm{K} / \mathrm{R})^{55}$, while $\mathrm{CARC}$ is oriented in the opposite direction $(\mathrm{K} / \mathrm{R})-\mathrm{X}_{1-5^{-}}(\mathrm{Y} / \mathrm{F} / \mathrm{W})-\mathrm{X}_{1-5^{-}}(\mathrm{L} / \mathrm{V})^{56}$. The cholesterol consensus motif $(\mathrm{CCM})$ is characterized by interactions with $(\mathrm{W} / \mathrm{Y})-(\mathrm{I} / \mathrm{V} / \mathrm{L})-(\mathrm{K} / \mathrm{R})$ and $(\mathrm{F} / \mathrm{Y} / \mathrm{R})^{54}$. By contrast, structures of membrane proteins (e.g., GPCRs and ion channels) determined in the presence of cholesterol/CHS have revealed interactions with "greasy hollows", which refers to pockets in the transmembrane portion lined with hydrophobic residues ${ }^{30,57,58}$.

Docking studies and MD simulations identified putative cholesterol binding sites in GIRK channels that involved various amino acid residues ${ }^{14,28,37}$. In particular, two sites are very similar to the positions observed in the cryoEM structure (CHS1 and CHS2). The model for the first site implicated several residues involved in the CHS1 hydrophobic pocket, including L95, V99, and $1175^{14}$. Additionally, the functional data suggest that V99 is involved in cholesterol binding. On the other hand, the second site is similar to the CHS2 pocket, as shown by functional and 
mutagenesis studies of several residues, including $\mathrm{L} 86$ and $\mathrm{V} 101^{14}$. Thus, the putative cholesterol binding sites identified in our cryoEM structures are supported by results from previous mutagenesis studies.

\section{Mechanism of $P_{2} P_{2}$ gating in GIRK2 channels}

Unexpectedly, we found the CTD is detached from the TMD in both the apo GIRK2 and a significant population of GIRK2 $2^{P I P 2}$ particles. By contrast, the CTD is engaged with the TMD in the structure of apo GIRK2 solved by X-ray crystallography ${ }^{23}$. One possibility for this difference could be due to lattice packing in X-ray crystal structures. Consistent with this idea, the CTD in the cryoEM structure of GIRK2 $2^{\mathrm{PIP} 2 *}$ is $\sim 1 \AA$ lower from the membrane than in the X-ray structure of GIRK2 $2^{P I P 2}$ (PDBID:3SYA, Extended Fig.S7a). A detached CTD in the absence of PIP 2 and other proteins (e.g. G proteins, SUR) may be a common feature of inward rectifiers, since the CTD is also detached in the apo Kir2.2 X-ray crystal structure ${ }^{26}$. Our cryoEM results suggest a plausible model for gating transitions in GIRK2 channels. First, $\mathrm{PIP}_{2}$ is clearly necessary for channel activation ${ }^{13,38}$. Second, the inter-domain region and the "wobbling" CTD are stabilized by the binding of four $\mathrm{PIP}_{2}$ molecules at the TMD-CTD interface, thus repositioning the stretched tether helix into a helical conformation and reducing the distance between Ca atoms of I195 and Q322 by 5-10 $\AA$ (Fig. 4). PIP 2 most likely serves a crucial role in stabilizing the CTD interaction with the TMD region and promoting activation, which could explain why GIRK channels are not gated open by $\mathrm{G} \beta \gamma$, ethanol or cholesterol in the absence of $\mathrm{PIP}_{2}$.

In addition to the detached CTD, there are also changes in the coordination of $\mathrm{PIP}_{2}$ with GIRK2 between the cryoEM structures (GIRK2 $2^{\mathrm{PIP} 2 / \mathrm{CHS}}$ and $\left.\mathrm{GIRK} 2^{\mathrm{PIP} 2 *}\right)$ and the X-ray crystal structure (PDBID:3SYA). The $1^{\prime} \mathrm{PO}_{4}$ of $\mathrm{PIP}_{2}$ in the cryoEM structures interacts with the side chain of R92, whereas in the X-ray crystal structure it is coordinated by the amide backbone of R92 and the side chain is not resolved. This difference might be explained by the lower CTD in the cryoEM 
structures (Extended Fig. 7a). In addition, the 4' $\mathrm{PO}_{4}$ interaction with $\mathrm{K} 64$ in $3 S \mathrm{SA}$ is not evident in the cryoEM structures. Interestingly, this pattern of engagement of $\mathrm{PIP}_{2}$ is similar to that observed with MD simulations of a constitutively open GIRK2 channel (K200Y $)^{59}$, raising the possibility that the cryoEM structures of GIRK2 are closer to an open state. In support of this, the position of phosphate atom of $1^{\prime} \mathrm{PO}_{4}$ of $\mathrm{PIP}_{2}$ is moved from the membrane towards cytoplasmic side by $3 \AA$ and accompanied with $\sim 3^{\circ}$ rotation of the CTD, resulting in a wider pore opening at the inner-helix gate in the GIRK2 ${ }^{\text {IIP2* }}$ cryoEM structure, as compared to the crystal structure (Extended Fig. S7).

\section{Role of cholesterol on $\mathrm{PIP}_{2}$ mediated channel gating}

Our cryoEM studies revealed one predominant conformation of GIRK2 ${ }^{\mathrm{PIP} 2 / \mathrm{CHS}}$, whereas several additional conformers with detached CTDs were observed in the absence of CHS, suggesting that CHS may have a role in stabilizing the $\mathrm{PIP}_{2}$-bound GIRK2 with engaged CTD (Fig. 5). These additional conformers of GIRK2 $2^{\text {PIP2 }}$ are likely due to the absence of cholesterol stabilization. Consistent with this observation, cholesterol stabilization of conformational states has been observed for several membrane proteins, in particular GPCRs, where the presence of cholesterol increases the thermal stability of the receptor and often lowers the energy barrier for agonist-induced conformational changes ${ }^{60}$. In addition, the binding of cholesterol in peripheral hydrophobic sites with no highly specific interactions is in agreement with the lack of large conformational changes on GIRK2 as a result of this engagement, implying its importance for primarily enhancing $\mathrm{PIP}_{2}$ binding. The increase in $\mathrm{PIP}_{2}$ affinity, as promoted by cholesterol, might increase the probability of the channel entering the open state. There is evidence that other allosteric modulators of channel activity such as alcohol and G proteins also act via increased $\mathrm{PIP}_{2}$ affinity ${ }^{61}$. 
GIRK2 is relatively unique among members of the Kir family because it is potentiated in response to cholesterol rather than inhibited, as occurs with most other members of the Kir family ${ }^{15}$. As such, it presents a unique therapeutic target as well as a powerful model system to probe the gating mechanisms of Kir channel family members. The identification of cholesterol docking sites by structural studies allows us to probe the primary determinants of its interactions and integrate it with the results of computational docking, MD simulations and site-directed mutagenesis. Additionally, small molecules that weaken or strengthen the $\mathrm{PIP}_{2}$ affinity, potentially through regulating cholesterol binding, could be employed to modulate GIRK channel function in a manner tuned to respond to specific pathology.

Acknowledgements: This work was supported in part by the National Institute on Alcohol Abuse and Alcoholism (AA018734) to PAS. We thank the Slesinger and Skiniotis laboratories for discussions on the experiments and E. Montabana for help with cryoEM data collection.

Author Contributions: YKM, IWG, GS and PAS contributed to the design of the experiments; IWG and YZ prepared protein and IWG conducted functional studies; YKM prepared vitrified samples, collected and processed cryoEM data, and performed modeling. MJR implemented GemSpot; YKM and GS analyzed EM data; YKM, IWG, GS and PAS interpreted the results and wrote the manuscript

Competing Interests statement. None for YKM, IWG, YZ, MJR, GW or PAS. 


\section{ON-LINE METHODS}

\section{Molecular Biology}

A truncated Mus musculus GIRK2 cDNA (containing amino acids 52-380) linked in-frame with an HRV 3C protease site, green fluorescent protein (GFP) and a decahistidine (HIS10) tag (a generous gift from R. MacKinnon, The Rockefeller University, New York, NY) in pPICZ (ThermoFisher) was transformed into Pichia pastoris using electroporation (according to manufacturer protocols). Transformants were screened based upon Zeocin resistance (>1 $\mathrm{mg} / \mathrm{ml}$ ) and GFP emission. Highest expressing clones were selected for large-scale purification.

\section{Protein purification and reconstitution}

All GIRK channels were expressed and purified in $P$. pastoris as described previously ${ }^{13}$. Briefly, the highest-expressing clone was grown in BMGY medium and induced in BMM medium containing $1 \%$ methanol. Cells were harvested, resuspended in buffer (50 mM HEPES, pH 7.4; $150 \mathrm{mM} \mathrm{KCl} ; 1 \mathrm{mM}$ TCEP; $1 \mathrm{mM} \mathrm{AEBSF}$ and Complete EDTA-free protease inhibitor tablets (Roche), flash frozen in liquid nitrogen, and stored at $-80^{\circ} \mathrm{C}$. Frozen cells were lysed in a Mixer Mill (Retsch) 5-times for 3 minutes at $25 \mathrm{~Hz}$ and stored as powder at $-80{ }^{\circ} \mathrm{C}$ until needed. The cell powder was solubilized in buffer containing $50 \mathrm{mM}$ HEPES, $\mathrm{pH} 7.35 ; 150 \mathrm{mM} \mathrm{KCl} ; 1 \mathrm{mM}$ TCEP; $1 \mathrm{mM}$ AEBSF; Complete ULTRA EDTA-free protease inhibitor tablets (Roche) and either $2 \%(w / v)$ n-Dodecyl- $\beta$-D-maltoside (DDM; Anatrace) or $2 \%(w / v)$ DDM supplemented with $0.2 \%$ (w/v) Cholesteryl Hemisuccinate Tris Salt (Anatrace) with gentle stirring at $4{ }^{\circ} \mathrm{C}$. Unsolubilized material was separated by centrifugation at $40,000 \times \mathrm{g}$ for $40 \mathrm{~min}$ at $4{ }^{\circ} \mathrm{C}$ and filtered. The supernatant was incubated with HISPur Cobalt charged resin (ThermoFisher) equilibrated in wash buffer (50 mM HEPES, pH 7.0; $150 \mathrm{mM} \mathrm{KCl;} \mathrm{0.2 \%} \mathrm{DDM} \mathrm{or} \mathrm{0.2 \% DDM/0.02 \%} \mathrm{CHS;} 20$ $\mathrm{mM}$ imidazole). The resin was subsequently washed in 10 column volumes (CV) of wash buffer, $5 \mathrm{CV}$ containing $40 \mathrm{mM}$ Imidazole, and eluted in buffer containing $300 \mathrm{mM}$ imidazole. The eluate was pooled, exchanged into imidazole-free buffer and digested overnight at $4^{\circ} \mathrm{C}$ with $\mathrm{HRV} 3 \mathrm{C}$ 
protease, purified as described ${ }^{62}$ (a generous gift of Daniel Minor, UCSF, San Francisco, CA). The protein was subsequently concentrated and run on a Superdex-200 gel filtration column in buffer containing $20 \mathrm{mM}$ TRIS-HCl pH 7.5, $150 \mathrm{mM} \mathrm{KCl,} 20 \mathrm{mM}$ DTT, $3 \mathrm{mM}$ TCEP, and $1 \mathrm{mM}$ EDTA, $0.025 \%(w / v)$ DDM (anagrade) alone or with $0.0025 \% \mathrm{CHS}$. Fractions eluting at a volume consistent with the GIRK channel tetramer were pooled, concentrated and examined by SDS-PAGE and Coomassie blue staining.

Purified GIRK2 channels were reconstituted into lipid vesicles as described previously ${ }^{13}$. Briefly, a lipid mixture containing 1-palmitoyl-2-oleoyl-sn-glycero-3-phosphoethanolamine (POPE), 1palmitoyl-2-oleoyl-sn-glycero-3-phospho-(1'-rac-glycerol) (POPG), and L- $\quad$ phosphatidylinositol-4,5-bisphosphate (Brain, $\mathrm{PI}(4,5) \mathrm{P}_{2}$ (Porcine)) at mass ratios of 3:1:0.04 (POPE:POPG:PIP ${ }_{2}$ ) or 3:1 (POPE:POPG) was prepared, reconstituted in vesicle buffer (20 mM K-HEPES, pH 7.4; 150 mM KCl; 0.5 mM EDTA containing 35 mM CHAPS) and incubated with protein in detergent at a 1:200 protein: lipid ratio unless otherwise indicated. Where indicated, cholesterol (Ovine wool; Anatrace) was added to vesicles at a mole percentage of $5 \%$ or Cholesteryl Hemisuccinate (CHS; Anatrace) was incorporated at $2.5 \%$ mol percentage. Detergent was removed through sequential addition of Bio-beads SM-2 (Bio-rad). All phospholipids, cholesterol, and Brain $\mathrm{PIP}_{2}$ were purchased from Avanti Polar Lipids, Inc. Watersoluble $\mathrm{PIP}_{2}\left(\mathrm{diC}_{8}-\mathrm{PIP}_{2}\right)$ was purchased from Echelon Biosciences.

\section{Flux assay}

The fluorescence-based flux assay for GIRK channel activity was performed as described previously ${ }^{13,18}$. Briefly, Liposomes were diluted 1:20 into flux buffer (20 mM Na-HEPES, pH 7.4; $150 \mathrm{mM} \mathrm{NaCl}$; $0.5 \mathrm{mM}$ EDTA) containing $5 \mu \mathrm{M}$ of the $\mathrm{H}^{+}$sensitive dye 9-Amino-6-chloro-2methoxyacridine (ACMA)(Invitrogen). Fluorescence was measured using a Flexstation 3 microplate reader (Molecular Devices) with the following parameters: $410 \mathrm{~nm}$ excitation, $480 \mathrm{~nm}$ emission, $455 \mathrm{~nm}$ cutoff, medium PMT sensitivity, and sampling at 2 seconds. After a stable 
baseline fluorescence (150 s) was obtained, the $\mathrm{H}^{+}$ionophore $\boldsymbol{m}$-chlorophenyl hydrazone (CCCP)(Sigma) was automatically added (1 $\mu \mathrm{M}$ final), then a second addition of vehicle or methanethiosulfonate hydroxyethyl (100 $\mu \mathrm{M}$ final; MTS-HE, Toronto Research Chemicals) was added $150 \mathrm{~s}$ later, followed $900 \mathrm{~s}$ later by a third addition with the $\mathrm{K}^{+}$ionophore Valinomycin (100 nM final; Invitrogen), to determine the maximal K+ flux. GIRK2 channels are likely arranged in both orientations in the liposomes. However, we expect the channels oriented inside-out to support high $\mathrm{K}^{+}$flux because of high $\mathrm{Na}^{+}$in the flux buffer and high $\mathrm{K}^{+}$in the liposome ${ }^{13}$. The percentage relative $\mathrm{K}^{+}$flux was calculated by measuring the extent of quenching $10 \mathrm{~s}$ before $\mathrm{VM}$ addition, and dividing by the total quenching capacity of the liposomes normalized to the relative fluorescence units (RFU) $10 \mathrm{~s}$ before the addition of vehicle or MTS-HE. Liposome flux assay illustration created with Biorender.com

\section{CryoEM sample preparation, data collection and processing}

The quality of purified samples was initially screened by negative stain EM using established protocols $^{39}$. For cryoEM, $3.5 \mu$ l of GIRK2 at a concentration of $7-10 \mathrm{mg} / \mathrm{ml}$ was applied to glowdischarged Quantifoil Au1.2/1.3, 200 mesh grids, blotted and then plunge-frozen in liquid ethane using FEI Vitrobot. All cryoEM data were collected at $300 \mathrm{kV}$ on a Titan Krios equipped with a Gatan K3 direct detection camera. Raw images were collected as movies, recorded at a magnification of 58,824 X corresponding to 0.85 pixel per $\AA$ at the specimen level. Each movie was recorded for $3 \mathrm{sec}$ at $0.05 \mathrm{sec} /$ frame with a total dose of 60 electrons/pixel and defocus values ranging from -0.8 to $-2.2 \mu \mathrm{m}$.

The movies were motion-corrected and dose-weighted using Motioncor $2^{63}$ and defocus values were determined by CTFFIND4 ${ }^{64}$. Template-based particle picking, 2D/3D classification, and 3D refinements were performed using the Relion pipeline ${ }^{65-68}$. The 3D classes with both domains distinguishable were refined with $\mathrm{C} 4$ or $\mathrm{C} 1$ symmetry as shown in Extended Figures S2, S4 and S6, post processed with a mask encompassing both the domains, corrected for modulation 
transfer function (MTF) of K3 direct detection camera at $300 \mathrm{kV}$ and sharpened with suitable B factor (Extended Table S1 and S2). The resolution of the maps reported here was estimated according to the 0.143 "gold-standard" Fourier Shell Correlation (FSC) criterion. The resolution of $\mathrm{GIRK}^{\mathrm{PIP} 2 / \mathrm{CHS}}, \mathrm{GIRK}^{\mathrm{PIP} 2 *}, \mathrm{GIRK} 2^{\mathrm{PIP} 2 * *}, \mathrm{GIRK}^{\mathrm{PIP} 2 * \star *}, \mathrm{GIRK} 2^{\mathrm{PIP} 2 * * *}$, and apo GIRK2 was indicated globally as $3.5,3.2,4.8,7.7,7.7$ and $4.8 \AA$, respectively. Local resolution was estimated using Relion. In apo GIRK2, the input particle stack for 3D classification was used for Relion multibody refinement analysis with TMD and CTD treated as separate bodies $^{69}$ (Extended Fig. S5e and Extended Movie S1).

\section{Modeling of protein and lipids in the cryoEM maps}

The GIRK2 backbone and $\mathrm{PIP}_{2}$ at the TMD-CTD interface as determined in the X-ray crystal structure of GIRK2 (PDBID:3SYA) was initially fit as rigid-body into the EM maps using UCSF Chimera $^{70}$ and subsequently interactively adjusted using COOT $^{41}$. The GemSpot pipeline ${ }^{40}$ was used for modeling CHS into EM map followed by refinement in $\mathrm{COOT}^{41}$. The models in the target EM maps were refined further using Phenix real-space refinement ${ }^{71}$. Pore dimension in the model was analyzed using the HOLE program ${ }^{72}$. The figures in the manuscript were prepared using UCSF ChimeraX ${ }^{73}$ 


\section{References}

1 Lüscher, C. \& Slesinger, P. A. Emerging roles for G protein-gated inwardly rectifying potassium (GIRK) channels in health and disease. Nat Rev Neurosci 11, 301-315 (2010).

2 Rifkin, R. A. et al. GIRK currents in VTA dopamine neurons control the sensitivity of mice to cocaine-induced locomotor sensitization. Proc. Natl. Acad. Sci. USA 115, E9479E9488, doi:10.1073/pnas.1807788115 (2018).

3 Munoz, M. B. \& Slesinger, P. A. Sorting nexin 27 regulation of G protein-gated inwardly rectifying $\mathrm{K}+$ channels attenuates in vivo cocaine response. Neuron 82, 659-669, doi:10.1016/j.neuron.2014.03.011 (2014).

4 Signorini, S., Liao, Y. J., Duncan, S. A., Jan, L. Y. \& Stoffel, M. Normal cerebellar development but susceptibility to seizures in mice lacking $\mathrm{G}$ protein-coupled, inwardly rectifying K+ channel GIRK2. Proc. Natl. Acad. Sci. USA 94, 923-927 (1997).

5 Hill, K. G., Alva, H., Blednov, Y. A. \& Cunningham, C. L. Reduced ethanol-induced conditioned taste aversion and conditioned place preference in GIRK2 null mutant mice. Psychopharmacology (Berl) 169, 108-114, doi:10.1007/s00213-003-1472-4 (2003).

6 Clarke, T. K. et al. KCNJ6 is associated with adult alcohol dependence and involved in gene $x$ early life stress interactions in adolescent alcohol drinking. Neuropsychopharm 36, 1142-1148, doi:10.1038/npp.2010.247 (2011).

7 Logothetis, D. E., Kurachi, Y., Galper, J., Neer, E. J. \& Clapham, D. E. The By subunits of GTP-binding proteins activate the muscarinic $\mathrm{K}^{+}$channel in heart. Nature 325, 321326 (1987).

8 Wickman, K. D. et al. Recombinant G-protein $\beta \gamma$-subunits activate the muscarinic-gated atrial potassium channel. Nature 368, 255-257 (1994).

9 Reuveny, E. et al. Activation of the cloned muscarinic potassium channel by G protein $\beta y$ subunits. Nature 370, 143-146 (1994).

10 Kobayashi, T. et al. Ethanol opens G-protein-activated inwardly rectifying $\mathrm{K}^{+}$channels. Nat. Neurosci. 2, 1091-1097 (1999).

11 Aryal, P., Dvir, H., Choe, S. \& Slesinger, P. A. A discrete alcohol pocket involved in GIRK channel activation. Nat Neurosci 12, 988-995, doi:nn.2358 [pii], 10.1038/nn.2358 (2009).

12 Lewohl, J. M. et al. G-protein-coupled inwardly rectifying potassium channels are targets of alcohol action. Nat. Neurosci. 2, 1084-1090 (1999). 
13 Glaaser, I. W. \& Slesinger, P. A. Dual activation of neuronal G protein-gated inwardly rectifying potassium (GIRK) channels by cholesterol and alcohol. Sci Rep 7, 4592, doi:10.1038/s41598-017-04681-x (2017).

14 Bukiya, A. N., Durdagi, S., Noskov, S. \& Rosenhouse-Dantsker, A. Cholesterol upregulates neuronal $G$ protein-gated inwardly rectifying potassium (GIRK) channel activity in the hippocampus. J. Biol. Chem. 292, 6135-6147, doi:10.1074/jbc.M116.753350 (2017).

15 Rosenhouse-Dantsker, A. Cholesterol Binding Sites in Inwardly Rectifying Potassium Channels. Adv Exp Med Biol 1135, 119-138, doi:10.1007/978-3-030-14265-0_7 (2019).

16 Bukiya, A. N. \& Dopico, A. M. Regulation of BK Channel Activity by Cholesterol and Its Derivatives. Adv Exp Med Biol 1115, 53-75, doi:10.1007/978-3-030-04278-3_3 (2019).

17 Finley, M., Arrabit, C., Fowler, C., Suen, K. F. \& Slesinger, P. A. bL-bM loop in the Cterminal domain of GIRK channels is important for Gßy activation. J. Physiol. 555, 643657 (2004).

18 Whorton, M. R. \& MacKinnon, R. X-ray structure of the mammalian GIRK2- $\beta$ Y G-protein complex. Nature 498, 190-197, doi:10.1038/nature12241 (2013).

19 Ivanina, T. et al. Mapping the Gßy-binding sites in GIRK1 and GIRK2 subunits of the G protein-activated K+ channel. J. Biol. Chem. 278, 29174-29183 (2003).

$20 \mathrm{He}, \mathrm{C}$. et al. Identification of critical residues controlling G protein-gated inwardly rectifying $\mathrm{K}^{+}$channel activity through interactions with the $\beta$ y subunits of $\mathrm{G}$ proteins. J. Biol. Chem. 277, 6088-6096 (2002).

21 Pegan, S. et al. Cytoplasmic domain structures of Kir2.1 and Kir3.1 show sites for modulating gating and rectification. Nat Neurosci 8, 279-287 (2005).

22 Jin, $T$. et al. The $\beta y$ subunits of $\mathrm{G}$ proteins gate a $\mathrm{K}^{+}$channel by pivoted bending of a transmembrane segment. Mol Cell 10, 469-481. (2002).

23 Whorton, M. R. \& MacKinnon, R. Crystal structure of the mammalian GIRK2 $\mathrm{K}^{+}$channel and gating regulation by $\mathrm{G}$ proteins, $\mathrm{PIP}_{2}$, and sodium. Cell 147, 199-208, doi:10.1016/j.cell.2011.07.046 (2011).

24 Pegan, S., Arrabit, C., Slesinger, P. A. \& Choe, S. Andersen's syndrome mutation effects on the structure and assembly of the cytoplasmic domains of Kir2.1. Biochemistry 45, 8599-8606 (2006).

25 Lopes, C. M. et al. Alterations in conserved Kir channel-PIP2 interactions underlie channelopathies. Neuron 34, 933-944 (2002). 
26 Hansen, S. B., Tao, X. \& MacKinnon, R. Structural basis of PIP2 activation of the classical inward rectifier K+ channel Kir2.2. Nature 477, 495-498, doi:10.1038/nature10370 (2011).

27 Fantini, J., Epand, R. M. \& Barrantes, F. J. Cholesterol-Recognition Motifs in Membrane Proteins. Adv Exp Med Biol 1135, 3-25, doi:10.1007/978-3-030-14265-0_1 (2019).

28 Levitan, I., Singh, D. K. \& Rosenhouse-Dantsker, A. Cholesterol binding to ion channels. Frontiers in physiology 5, 65, doi:10.3389/fphys.2014.00065 (2014).

29 Genheden, S., Essex, J. W. \& Lee, A. G. G protein coupled receptor interactions with cholesterol deep in the membrane. Biochim Biophys Acta Biomembr 1859, 268-281, doi:10.1016/j.bbamem.2016.12.001 (2017).

30 Lee, A. G. Interfacial Binding Sites for Cholesterol on G Protein-Coupled Receptors. Biophys. J. 116, 1586-1597, doi:10.1016/j.bpj.2019.03.025 (2019).

31 Oates, J. \& Watts, A. Uncovering the intimate relationship between lipids, cholesterol and GPCR activation. Curr Opin Struct Biol 21, 802-807, doi:10.1016/j.sbi.2011.09.007 (2011).

32 Prasanna, X., Chattopadhyay, A. \& Sengupta, D. Cholesterol modulates the dimer interface of the beta(2)-adrenergic receptor via cholesterol occupancy sites. Biophys. J. 106, 1290-1300, doi:10.1016/j.bpj.2014.02.002 (2014).

33 Prasanna, X., Mohole, M., Chattopadhyay, A. \& Sengupta, D. Role of cholesterolmediated effects in GPCR heterodimers. Chem Phys Lipids 227, 104852, doi:10.1016/j.chemphyslip.2019.104852 (2020).

34 Epshtein, Y. et al. Identification of a C-terminus domain critical for the sensitivity of Kir2.1 to cholesterol. Proc. Natl. Acad. Sci. USA 106, 8055-8060, doi:10.1073/pnas.0809847106 (2009).

35 Rosenhouse-Dantsker, A., Leal-Pinto, E., Logothetis, D. E. \& Levitan, I. Comparative analysis of cholesterol sensitivity of Kir channels: role of the CD loop. Channels (Austin) 4, 63-66, doi:10.4161/chan.4.1.10366 (2010).

36 Rosenhouse-Dantsker, A., Noskov, S., Durdagi, S., Logothetis, D. E. \& Levitan, I. Identification of novel cholesterol-binding regions in Kir2 channels. J. Biol. Chem. 288, 31154-31164, doi:10.1074/jbc.M113.496117 (2013).

37 Duncan, A. L., Corey, R. A. \& Sansom, M. S. P. Defining how multiple lipid species interact with inward rectifier potassium (Kir2) channels. Proc Natl Acad Sci U S A 117, 7803-7813, doi:10.1073/pnas.1918387117 (2020). 
38 Huang, C. L., Feng, S. \& Hilgemann, D. W. Direct activation of inward rectifier potassium channels by PIP2 and its stabilization by Gbetagamma. Nature 391, 803-806, doi:10.1038/35882 (1998).

39 Peisley, A. \& Skiniotis, G. 2D Projection Analysis of GPCR Complexes by Negative Stain Electron Microscopy. Methods Mol Biol 1335, 29-38, doi:10.1007/978-1-4939-2914-6_3 (2015).

40 Robertson, M. J., van Zundert, G. C. P., Borrelli, K. \& Skiniotis, G. GemSpot: A Pipeline for Robust Modeling of Ligands into Cryo-EM Maps. Structure, doi:10.1016/j.str.2020.04.018 (2020).

41 Emsley, P. \& Cowtan, K. Coot: model-building tools for molecular graphics. Acta Crystallogr D Biol Crystallogr 60, 2126-2132, doi:10.1107/S0907444904019158 (2004).

42 Rosenson, R. S. et al. HDL and atherosclerotic cardiovascular disease: genetic insights into complex biology. Nat Rev Cardiol 15, 9-19, doi:10.1038/nrcardio.2017.115 (2018).

43 Dietschy, J. M. \& Turley, S. D. Cholesterol metabolism in the brain. Curr Opin Lipidol 12, 105-112 (2001).

44 Vance, J. E. Dysregulation of cholesterol balance in the brain: contribution to neurodegenerative diseases. Dis Model Mech 5, 746-755, doi:10.1242/dmm.010124 (2012).

45 Engel, D. F. et al. Is there an association between hypercholesterolemia and depression? Behavioral evidence from the LDLr(-/-) mouse experimental model. Behav Brain Res 311, 31-38, doi:10.1016/j.bbr.2016.05.029 (2016).

46 Paul, R. et al. Hypercholesterolemia causes psychomotor abnormalities in mice and alterations in cortico-striatal biogenic amine neurotransmitters: Relevance to Parkinson's disease. Neurochem. Int. 108, 15-26, doi:10.1016/j.neuint.2017.01.021 (2017).

47 Paul, R. et al. Cholesterol contributes to dopamine-neuronal loss in MPTP mouse model of Parkinson's disease: Involvement of mitochondrial dysfunctions and oxidative stress. PLoS One 12, e0171285, doi:10.1371/journal.pone.0171285 (2017).

48 Paul, R. et al. Accumulation of Cholesterol and Homocysteine in the Nigrostriatal Pathway of Brain Contributes to the Dopaminergic Neurodegeneration in Mice. Neuroscience 388, 347-356, doi:10.1016/j.neuroscience.2018.07.041 (2018).

49 Cho, Y. Y., Kwon, O. H., Park, M. K., Kim, T. W. \& Chung, S. Elevated cellular cholesterol in Familial Alzheimer's presenilin 1 mutation is associated with lipid raft localization of beta-amyloid precursor protein. PLoS One 14, e0210535, doi:10.1371/journal.pone.0210535 (2019). 
$50 \quad$ Zarrouk, A. et al. Lipid Biomarkers in Alzheimer's Disease. Curr Alzheimer Res 15, 303312, doi:10.2174/1567205014666170505101426 (2018).

51 Jones, O. T. \& McNamee, M. G. Annular and nonannular binding sites for cholesterol associated with the nicotinic acetylcholine receptor. Biochemistry 27, 2364-2374, doi:10.1021/bi00407a018 (1988).

52 Dreger, M., Krauss, M., Herrmann, A. \& Hucho, F. Interactions of the nicotinic acetylcholine receptor transmembrane segments with the lipid bilayer in native receptorrich membranes. Biochemistry 36, 839-847, doi:10.1021/bi960666z (1997).

53 Middlemas, D. S. \& Raftery, M. A. Identification of subunits of acetylcholine receptor that interact with a cholesterol photoaffinity probe. Biochemistry 26, 1219-1223, doi:10.1021/bi00379a003 (1987).

54 Hanson, M. A. et al. A specific cholesterol binding site is established by the $2.8 \mathrm{~A}$ structure of the human beta2-adrenergic receptor. Structure 16, 897-905, doi:10.1016/j.str.2008.05.001 (2008).

55 Li, H. \& Papadopoulos, V. Peripheral-type benzodiazepine receptor function in cholesterol transport. Identification of a putative cholesterol recognition/interaction amino acid sequence and consensus pattern. Endocrinology 139, 4991-4997, doi:10.1210/endo.139.12.6390 (1998).

56 Baier, C. J., Fantini, J. \& Barrantes, F. J. Disclosure of cholesterol recognition motifs in transmembrane domains of the human nicotinic acetylcholine receptor. Sci Rep 1, 69, doi:10.1038/srep00069 (2011).

57 Lee, A. G. Interfacial Binding Sites for Cholesterol on TRP Ion Channels. Biophys J 117, 2020-2033, doi:10.1016/j.bpj.2019.10.011 (2019).

58 Duncan, A. L., Song, W. \& Sansom, M. S. P. Lipid-Dependent Regulation of lon Channels and G Protein-Coupled Receptors: Insights from Structures and Simulations. Annu Rev Pharmacol Toxicol 60, 31-50, doi:10.1146/annurev-pharmtox-010919-023411 (2020).

59 Lacin, E. et al. Dynamic role of the tether helix in PIP2-dependent gating of a G proteingated potassium channel. J. Gen. Physiol. 149, 799-811, doi:10.1085/jgp.201711801 (2017).

60 Gimpl, G. Interaction of G protein coupled receptors and cholesterol. Chem Phys Lipids 199, 61-73, doi:10.1016/j.chemphyslip.2016.04.006 (2016).

61 Bodhinathan, K. \& Slesinger, P. A. Molecular mechanism underlying ethanol activation of G-protein-gated inwardly rectifying potassium channels. Proc. Natl. Acad. Sci. USA 110, 18309-18314, doi:10.1073/pnas.1311406110 (2013). 
62 Shaya, D. et al. Voltage-gated sodium channel ( $\mathrm{NaV})$ protein dissection creates a set of functional pore-only proteins. Proc Natl Acad Sci U S A 108, 12313-12318, doi:10.1073/pnas.1106811108 (2011).

63 Zheng, S. Q. et al. MotionCor2: anisotropic correction of beam-induced motion for improved cryo-electron microscopy. Nat Methods 14, 331-332, doi:10.1038/nmeth.4193 (2017).

64 Rohou, A. \& Grigorieff, N. CTFFIND4: Fast and accurate defocus estimation from electron micrographs. J Struct Biol 192, 216-221, doi:10.1016/j.jsb.2015.08.008 (2015).

65 Zivanov, J. et al. New tools for automated high-resolution cryo-EM structure determination in RELION-3. eLife 7, doi:10.7554/eLife.42166 (2018).

66 Scheres, S. H. RELION: implementation of a Bayesian approach to cryo-EM structure determination. J Struct Biol 180, 519-530, doi:10.1016/j.jsb.2012.09.006 (2012).

67 Scheres, S. H. Semi-automated selection of cryo-EM particles in RELION-1.3. J Struct Biol 189, 114-122, doi:10.1016/j.jsb.2014.11.010 (2015).

68 Scheres, S. H. Processing of Structurally Heterogeneous Cryo-EM Data in RELION. Methods Enzymol 579, 125-157, doi:10.1016/bs.mie.2016.04.012 (2016).

69 Nakane, T., Kimanius, D., Lindahl, E. \& Scheres, S. H. Characterisation of molecular motions in cryo-EM single-particle data by multi-body refinement in RELION. Elife 7, doi:10.7554/eLife.36861 (2018).

70 Pettersen, E. F. et al. UCSF Chimera--a visualization system for exploratory research and analysis. J Comput Chem 25, 1605-1612, doi:10.1002/jcc.20084 (2004).

71 Afonine, P. V. et al. Real-space refinement in PHENIX for cryo-EM and crystallography. Acta Crystallogr D Struct Biol 74, 531-544, doi:10.1107/S2059798318006551 (2018).

72 Smart, O. S., Neduvelil, J. G., Wang, X., Wallace, B. A. \& Sansom, M. S. HOLE: a program for the analysis of the pore dimensions of ion channel structural models. $J \mathrm{Mol}$ Graph 14, 354-360, 376, doi:10.1016/s0263-7855(97)00009-x (1996).

73 Goddard, T. D. et al. UCSF ChimeraX: Meeting modern challenges in visualization and analysis. Protein science : a publication of the Protein Society 27, 14-25, doi:10.1002/pro.3235 (2018). 
a

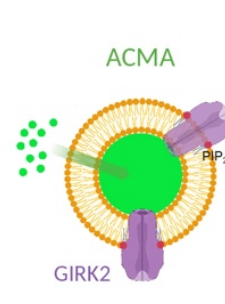

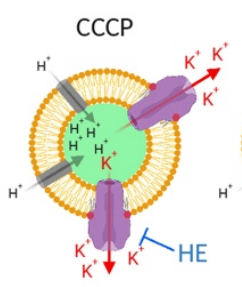

VM
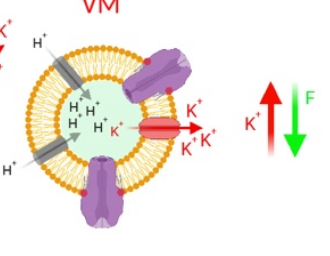

b
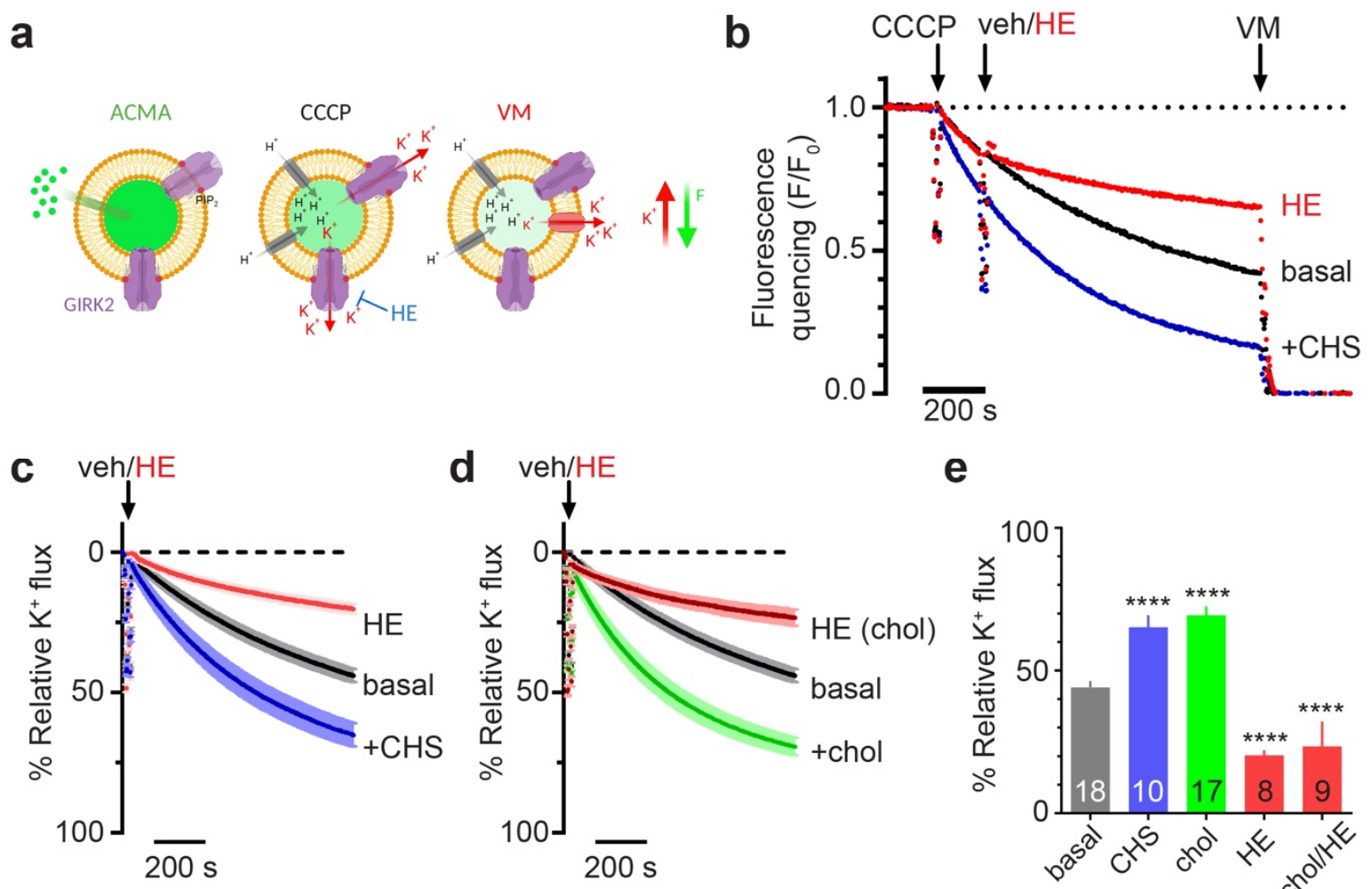

e

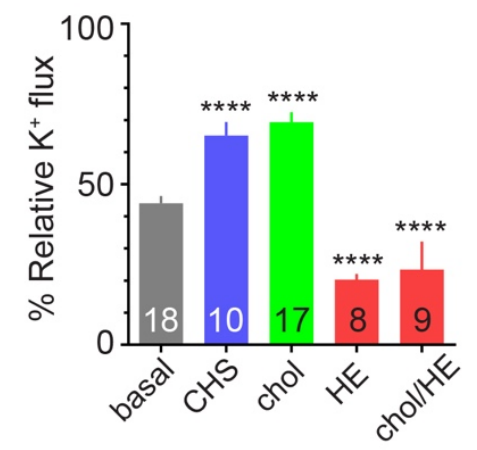

Figure 1: CHS potentiates GIRK2 activity in proteoliposomes with $\mathrm{PIP}_{2}$. (a) Schematic of the $\mathrm{K}^{+}$flux assay. Left panel indicates proteoliposomes pre-loaded with ACMA at baseline. Middle panel demonstrates fluorescence quenching in the presence of open GIRK channels upon addition of CCCP and $\mathrm{H}^{+}$influx. Right panel shows the dissipation of the $\mathrm{K}^{+}$gradient upon addition of valinomycin (VM), indicating the vesicle capacity. (b) Example of full-length normalized fluorescent traces of GIRK2containing liposomes with $1 \%$ brain $\mathrm{PIP}_{2}$. Fluorescence quenching is measured upon addition of CCCP (arrow), and then after addition of GIRK2 channel inhibitor $100 \mu \mathrm{M}$ MTS-HE (HE) or vehicle (veh, arrow), and then terminated by addition of VM. Note increase in quenching in the presence of CHS (blue trace), as compared to basal (black trace) and HE inhibited (red trace). (c) Plot shows normalized \% relative $\mathrm{K}^{+}$ flux (mean \pm SEM) of GIRK2-containing liposomes with $1 \%$ brain $\mathrm{PIP}_{2}+\mathrm{CHS}$ (blue trace, $\mathrm{n}=10$ ) or $1 \%$ brain $\mathrm{PIP}_{2}$ alone (basal; black trace, $\mathrm{n}=18$ ) following addition of veh, or after the addition of MTS-HE for $1 \%$ brain $\mathrm{PIP}_{2}$ alone (red trace, $\mathrm{n}=8$ ). (d) Normalized \% relative $\mathrm{K}^{+}$flux (mean $\pm \mathrm{SEM}$ ) of GIRK2containing liposomes with $1 \%$ brain $\mathrm{PIP}_{2}+5 \%$ cholesterol (green trace, $\mathrm{n}=17$ ) or $1 \%$ brain $\mathrm{PIP}_{2}$ alone (black, same as b) following addition of veh, or following addition of $\mathrm{HE}$ for $1 \%$ brain $\mathrm{PIP}_{2}+5 \%$ cholesterol (red trace, $n=9$ ). (e) Bar graph shows the average ( $\pm \square$ SEM; $n$ indicated on graph) \% relative $\mathrm{K}^{+}$flux for the indicated conditions. Both $\mathrm{CHS}$ and cholesterol significantly increased the $\%$ relative $\mathrm{K}^{+}$flux ${ }^{* * * *} p<0.0001$ vs. basal). HE (red bar) significantly inhibited GIRK2 channels. 
a

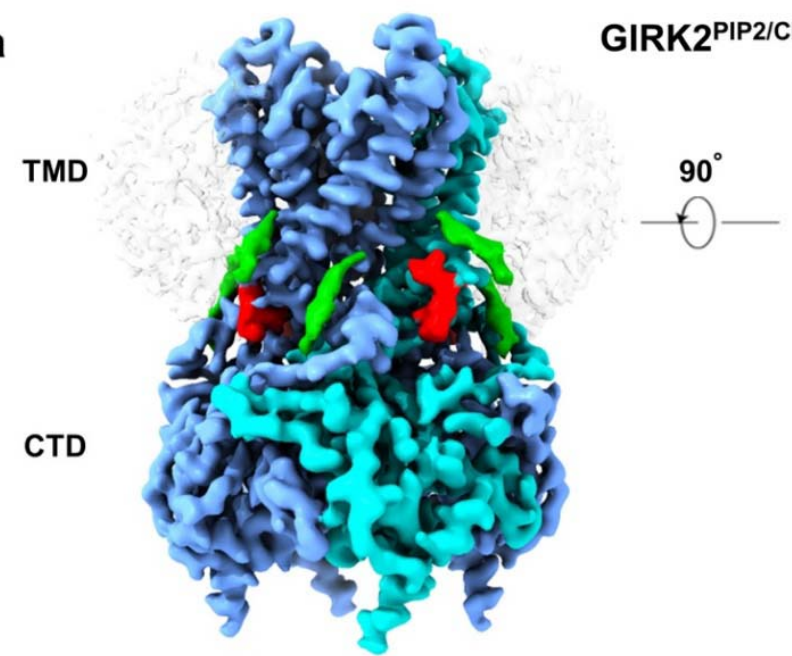

\section{GIRK2 ${ }^{\text {PIP2/CHS }}$}

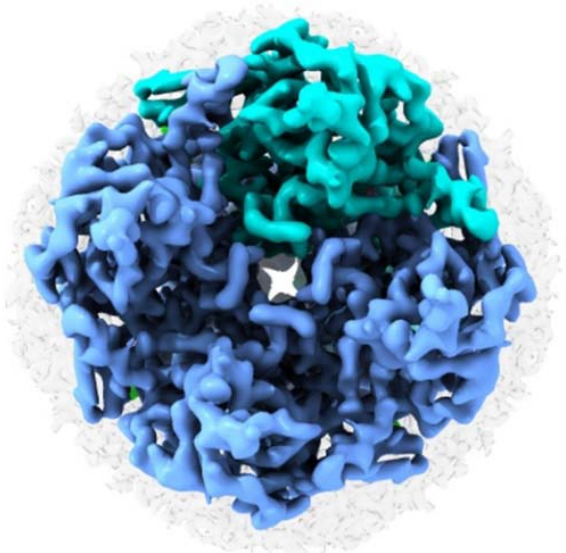

b
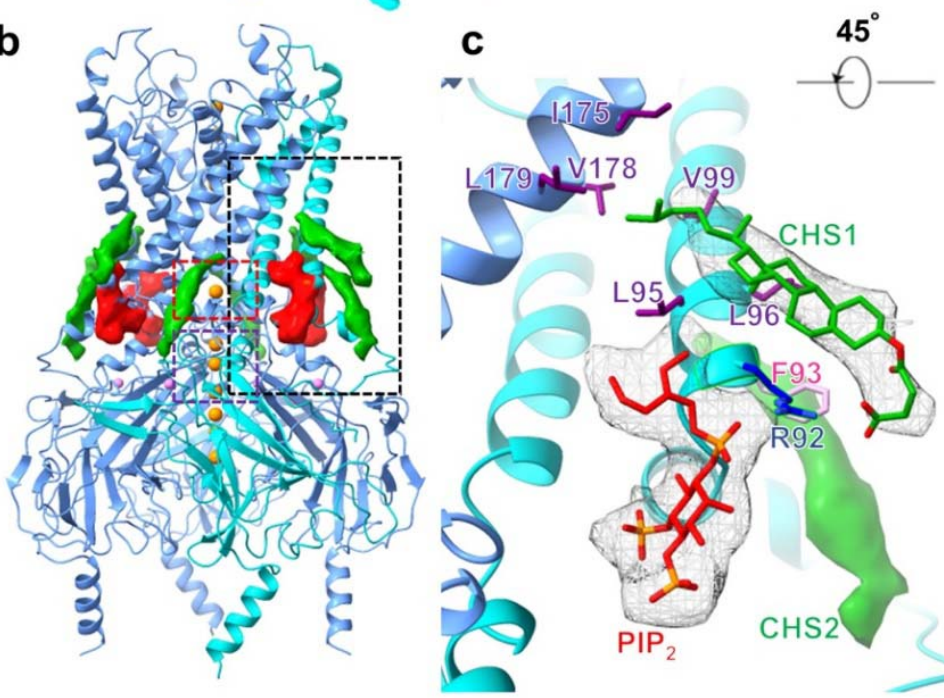

d

d
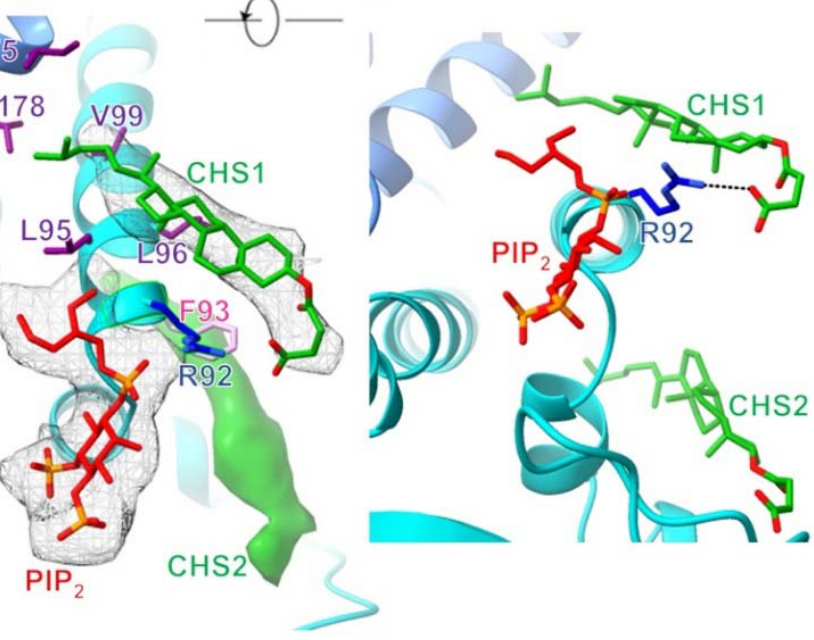

$\mathrm{PI}_{2}$

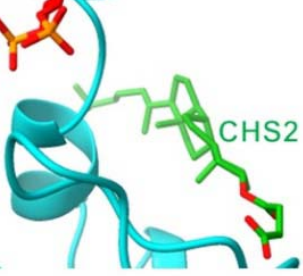

Figure 2: Structure of GIRK2 with $\mathrm{PIP}_{2}$ and cholesteryl hemisuccinate (CHS). (a) GIRK2 $2^{\mathrm{PI} 2 / \mathrm{CHS}}$ cryoEM map; two CHS (green) molecules per subunit are modelled into planar densities on the opposite side of $\mathrm{PIP}_{2}$ (red)-channel interface. GIRK2 subunits are in blue with one highlighted in cyan; DDM micelle is shown in transparency. The top right panel shows bottom-top view from cytoplasmic side. (b) Model of GIRK2 $2^{\mathrm{PI} 2 / \mathrm{CHS}}$ with the transmembrane and cytoplasmic domains. $\mathrm{K}^{+}$ions (orange) are shown along the pore and one $\mathrm{Na}^{+}$ion (violet) is modelled per subunit as seen in X-ray crystal structures. The densities of CHS and anionic head group of $\mathrm{PIP}_{2}$ are shown at the TMD-CTD interface. The inner-helix and G-loop gates are highlighted in red and purple dashed boxes, respectively, and observed CHS pockets is in black dashed box. (c) $\mathrm{CHS}$ (green, density shown as mesh, referred to as CHS1) modeled into a density near $\mathrm{PIP}_{2}$ (red, density shown as mesh) is surrounded by positively charged R92 (dark blue) and hydrophobic residues including F93 (pink), L95, L96, V99 (purple) from one protomer (cyan) and I175, V178, L179 (purple) from nearby subunit (blue). The density of other CHS molecule (green, referred to as CHS2) adjacent to this is represented as surface. The densities of anionic head group of $\mathrm{PIP}_{2}$ and $\mathrm{CHS}$ are contoured at a threshold level of 0.0073 in ChimeraX. (d) CHS1 is on the opposite side of $\mathrm{PIP}_{2}$ in the GIRK2 $2^{\mathrm{PI} 2 / \mathrm{CHS}}$ map and its carboxylate group coordinates with the side chain of R92 (dark blue). 


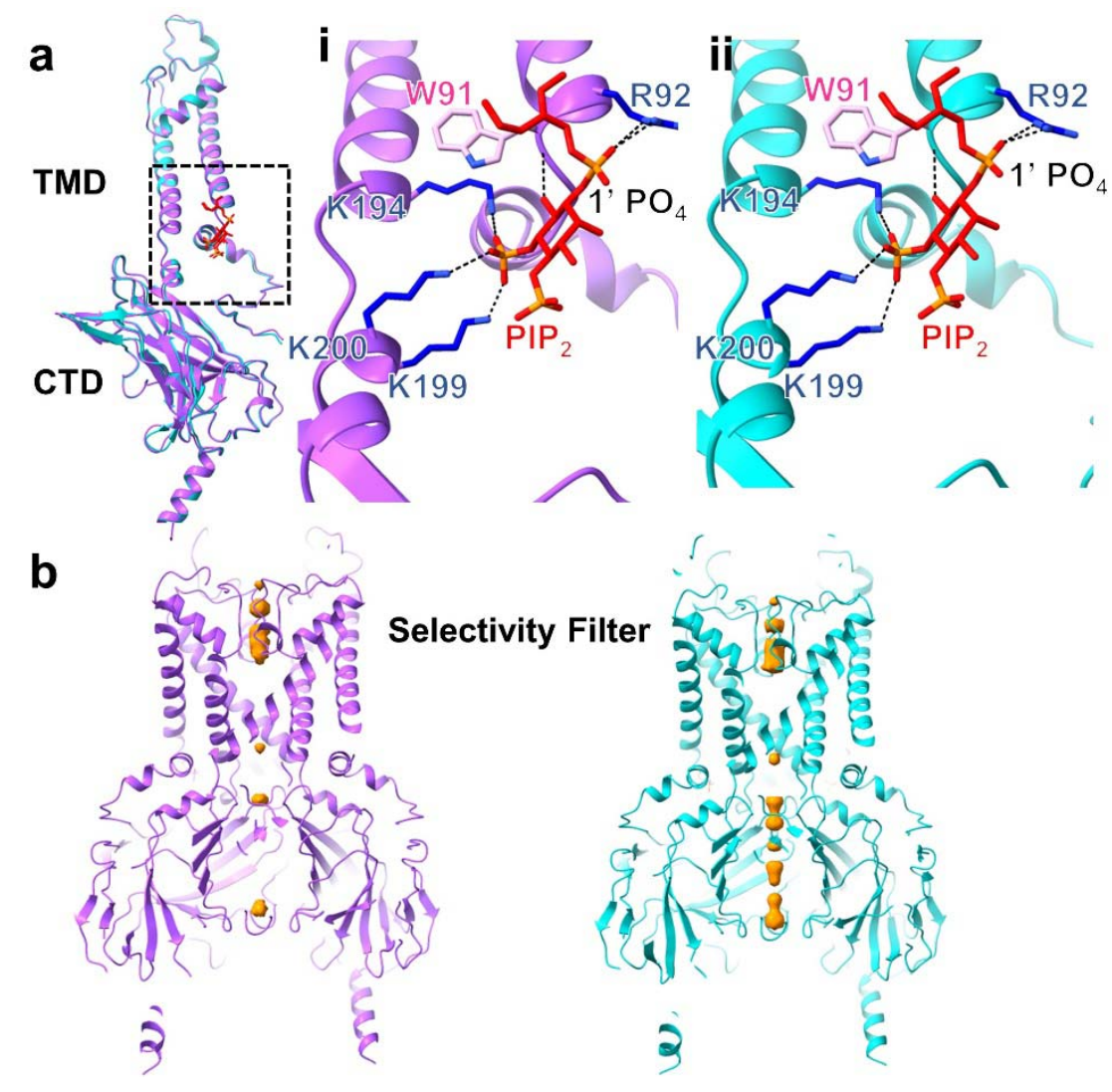

Figure 3: $\mathrm{PIP}_{2}$ binding pocket and $\mathrm{K}^{+}$ions present in cryoEM structures. (a) Comparison of a subunit from GIRK2 $2^{\mathrm{PIP} 2 *}$ (purple) and GIRK2 $2^{\mathrm{PIP} 2 / \mathrm{CHS}}$ (cyan) structures; the RMSD between them is around $0.4 \AA$. Insets (i) and (ii) represent $\mathrm{PIP}_{2}$ (red) coordination in GIRK2 ${ }^{\mathrm{PIP} 2 *}$ (purple) and GIRK2 ${ }^{\mathrm{PIP} 2 / \mathrm{CHS}}$ (cyan) respectively; $\mathrm{PIP}_{2}$ interacts with side chain of positively charged residues $\mathrm{R} 92, \mathrm{~K} 194, \mathrm{~K} 199$, K200 (dark blue) and amide backbone of W91 (pink). In cryoEM structures, the $1^{\prime} \mathrm{PO}_{4}$ of $\mathrm{PIP}_{2}$ is coordinated by the side chain of R92. (b) $\mathrm{K}^{+}$ions were modelled into densities in the pore of GIRK2 ${ }^{\mathrm{PIP}^{*} \text { (purple) and }}$ GIRK2 $^{\text {PIP2/CHS }}$ (cyan) cryoEM maps (orange, contoured at a threshold level of 0.0045 in ChimeraX). Apart from the selectivity filter, additional densities compatible with $\mathrm{K}^{+}$ions are observed along the pore. 

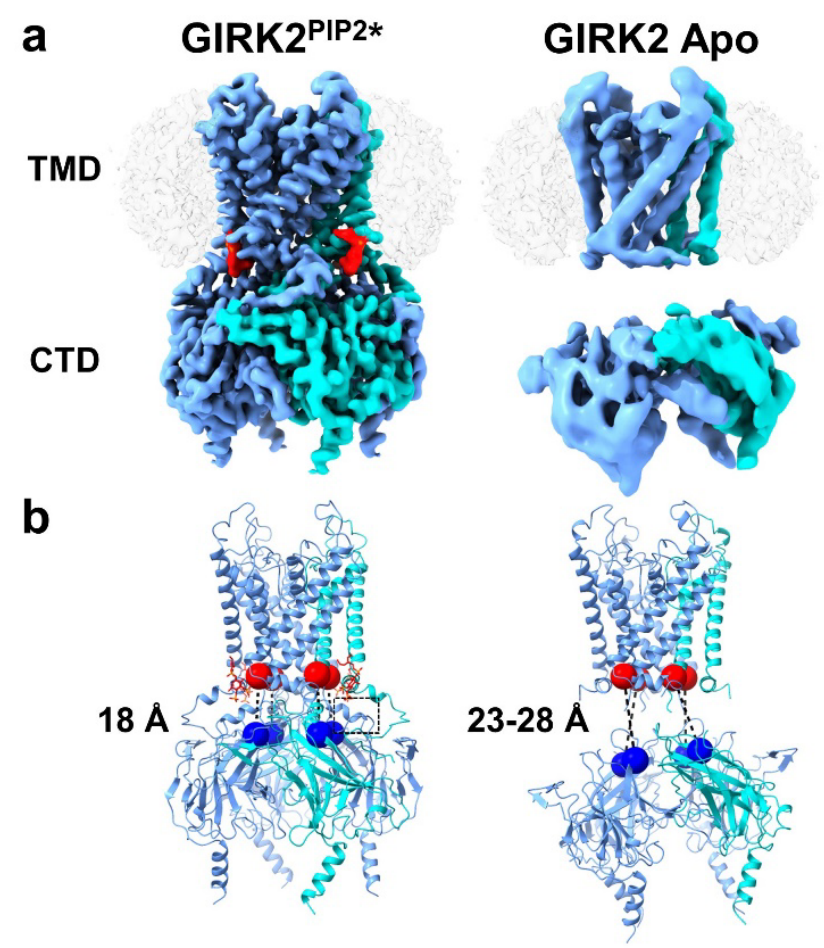

Figure 4: CryoEM structures of GIRK2 with and without PIP $_{2 .}$ (a) CryoEM maps of the most prevalent GIRK2 $^{\mathrm{PIP} 2 *}$ and GIRK2 apo conformations; the CTD is disengaged from membrane in apo compared to the GIRK2 $2^{\mathrm{PIP} 2 *}$ structure with the tether helix (residues 197-203) and N-terminal residues 67-78 disordered. PIP $_{2}$ (red) is at TMD-CTD interface, one subunit is highlighted in cyan and micelle is in transparency. (b) Evaluation of CTD detachment in GIRK2 ${ }^{\mathrm{PI} 2 *}$ and GIRK2 apo, the distance between Ca atoms of residues 1195 (denoted by red sphere) and Q322 (denoted by blue sphere) in these structures are 18 and 23-28 A, respectively. The ordered tether helix from one subunit of GIRK2 $2^{\mathrm{PI}^{*}}$ is shown with a dashed square. 


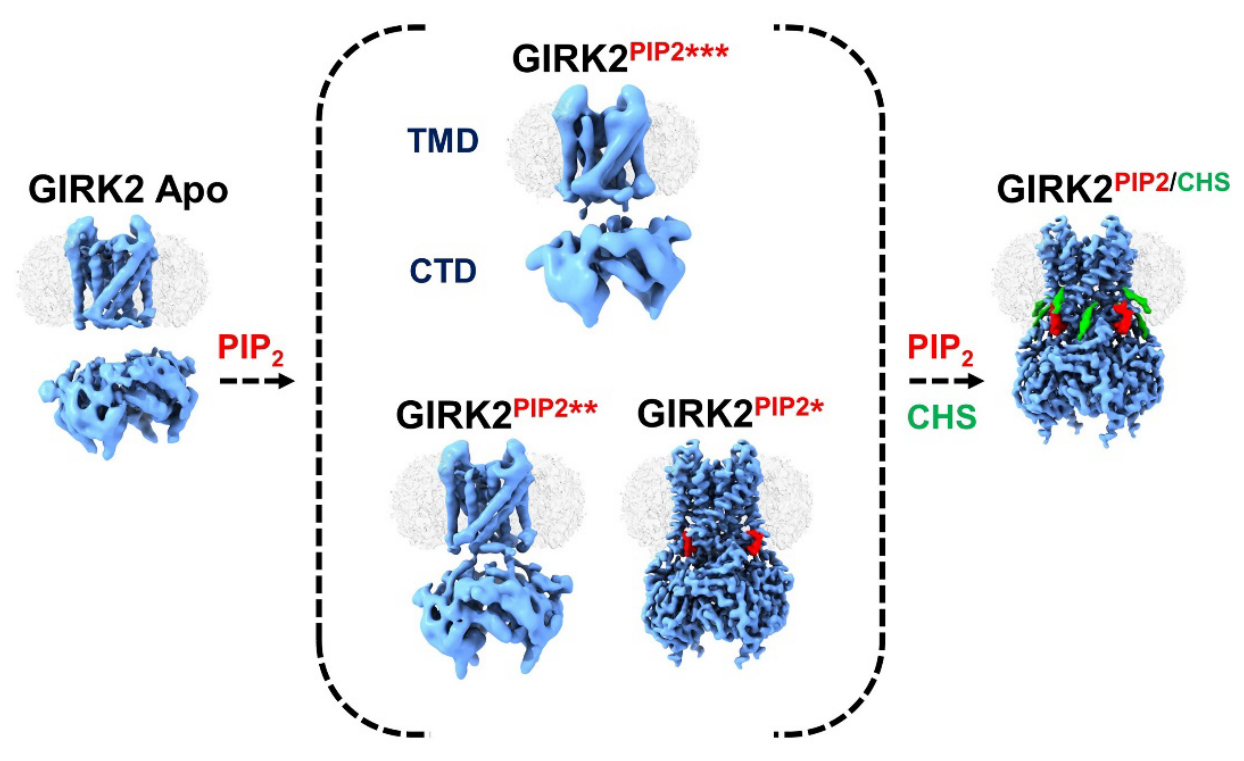

Figure 5: Different gating states for GIRK2 revealed by cryoEM. Structures with different lipid modulators suggest varying conformation of CTD with respect to TMD. The structures reveal novel cholesterol (green) binding sites adjacent to $\mathrm{PIP}_{2}$ (red) pocket. These molecules stabilize the TMD-CTD interface, thereby aiding in anchoring of CTD on to the membrane for further activation. The fraction of particles contributing to GIRK2 apo, GIRK2 $2^{\mathrm{PIP} 2 *}$, GIRK2 $2^{\mathrm{PIP} 2 * *}, \mathrm{GIRK}^{\mathrm{PIP} 2 * *}$ and $\mathrm{GIRK} 2^{\mathrm{PIP} 2 / \mathrm{CHS}}$ maps as obtained from the cryoEM data processing is $22,39,27,10$ and $62 \%$, respectively. 


\section{Extended Supplemental Figures:}
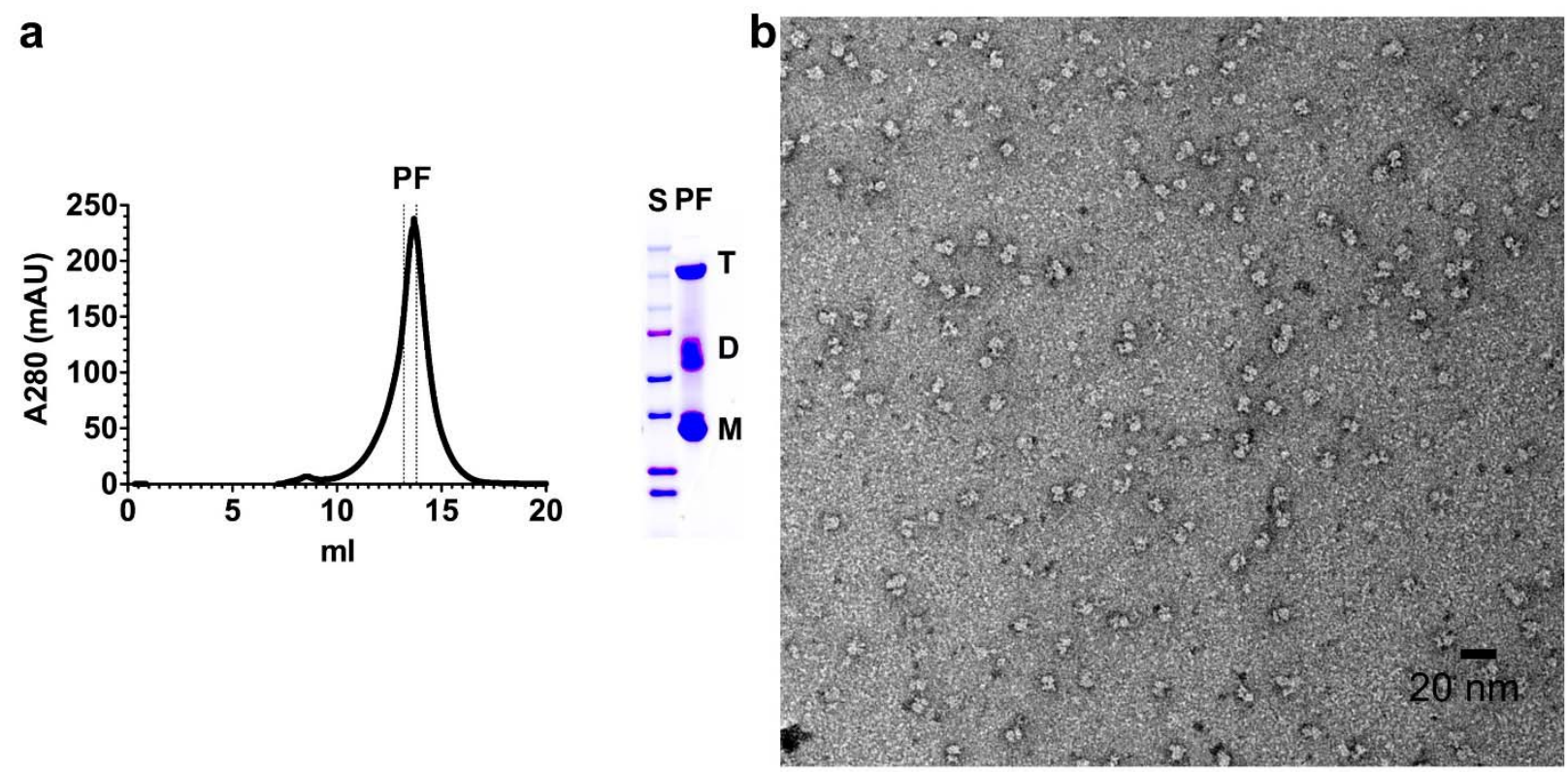

Extended Figure S1: Purification and assessment of sample quality by negative staining electron microscopy. (a) Size exclusion chromatogram and Coomassie staining of purified GIRK2. The chromatogram (left panel) shows purification of mouse GIRK2 from Pichia pastoris. The absorbance is plotted as a function of elution volume. The major peak elutes at a volume consistent with the size of a $\Delta$ GIRK2 tetramer'. Peak fractions (PF) utilized in both functional and structural studies are indicated by dashed vertical lines. Coomassie blue staining (right panel) of pooled and concentrated peak fractions (PF) on a gradient SDS-PAGE protein gel shows three bands, one for tetramer (T), one for dimer (D), and one for monomer (M). (b) Micrograph of negative stained GIRK2. The image shows predominantly homogenous GIRK2 tetramers in detergent micelle. 

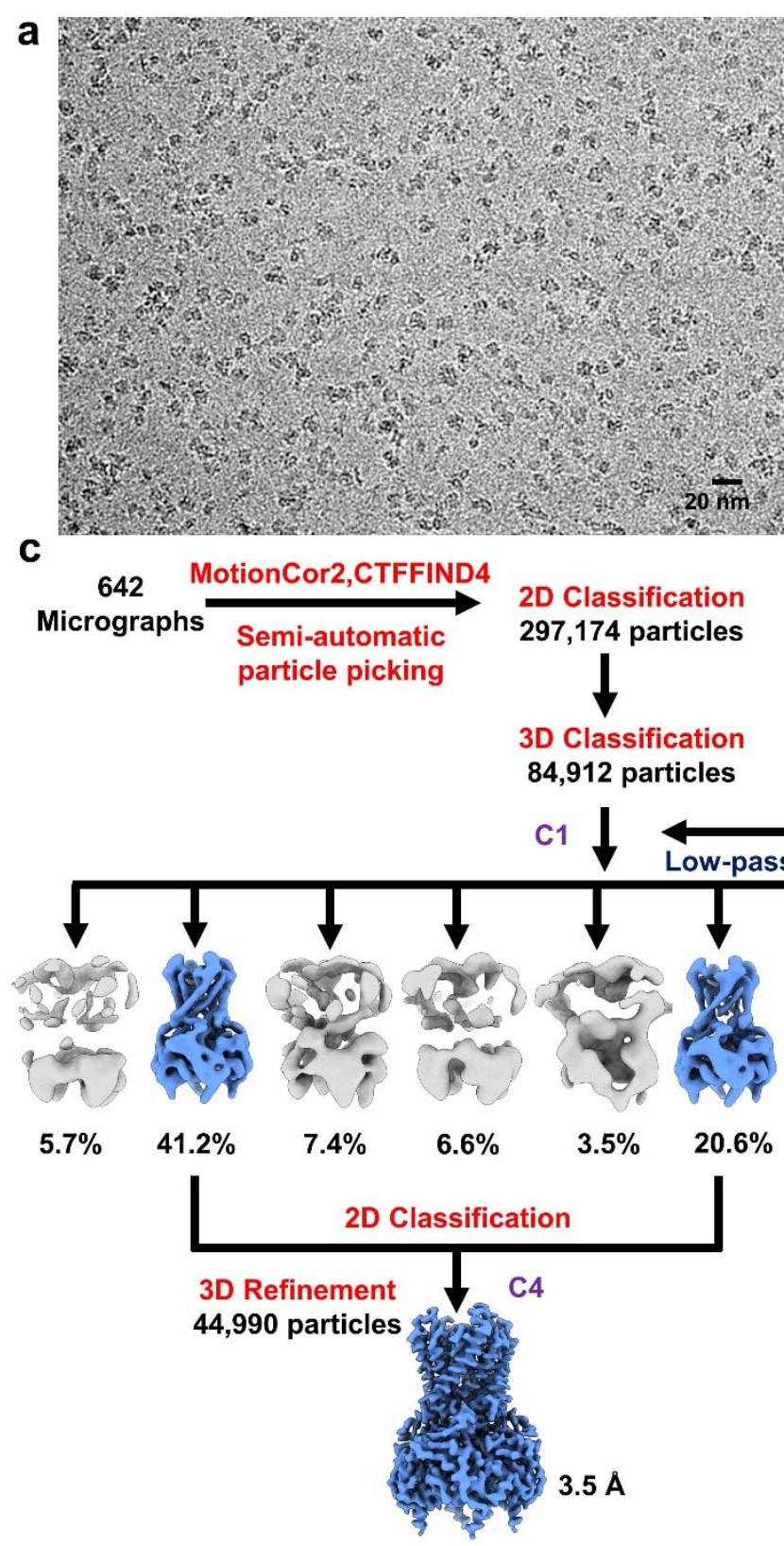

b

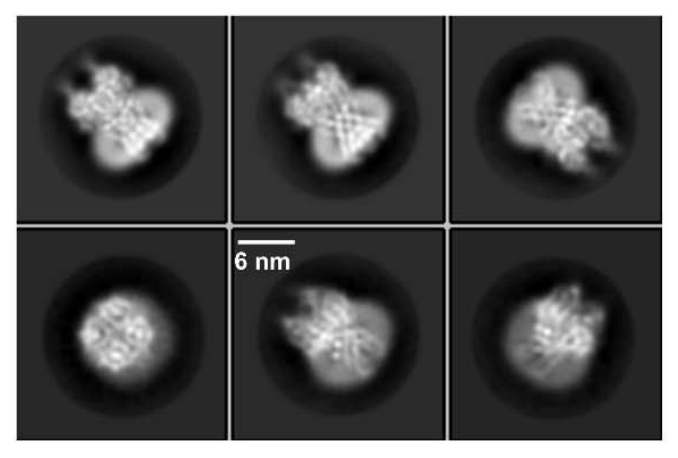

$20 \mathrm{hm}$.

2D Classification

297,174 particles

$\checkmark$

model

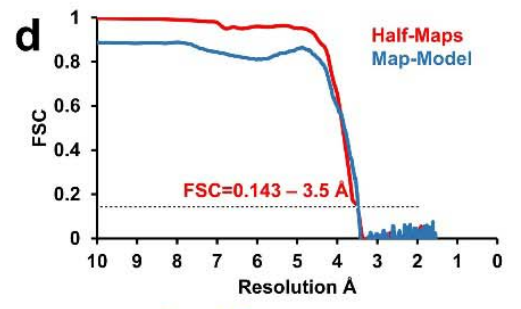

e

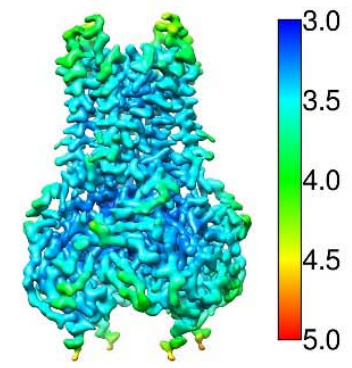

$7.5 \%$

Extended Figure S2: CryoEM data processing of GIRK2 in presence of modulators PIP $_{2}$ and cholesteryl hemisuccinate. (a) and (b) are representative cryoEM image and 2D averages. (c) Data processing flow chart. (d) FSC curve, the dotted line indicating FSC at 0.143. (e) Local resolution estimate by Relion. (f) Fit of the transmembrane helices M1 and M2 into the $3.5 \AA$ GIRK2 ${ }^{\text {IIP2/CHS }}$ cryoEM map. 


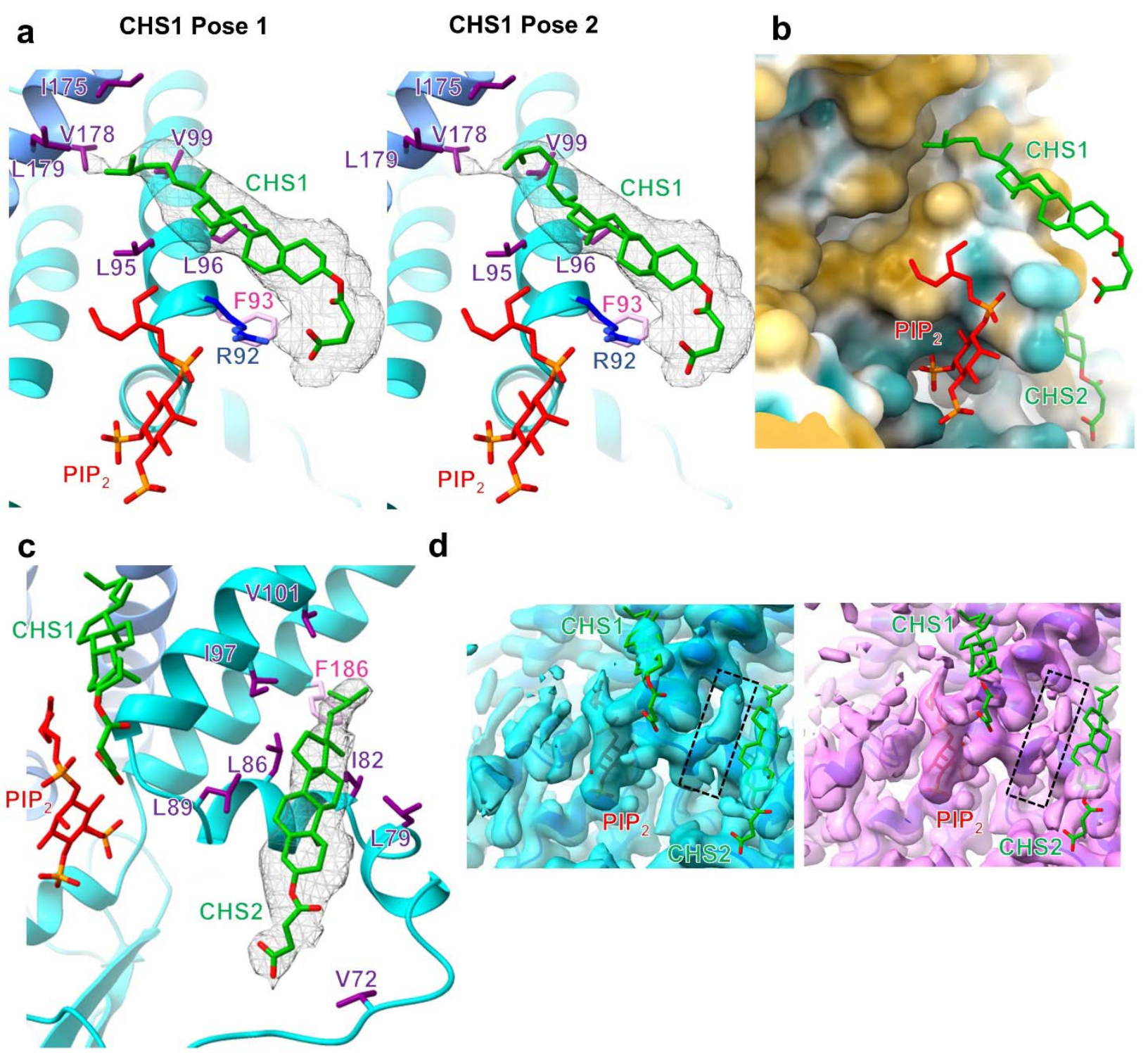

Extended Figure S3: Modeling of cholesteryl hemisuccinate (CHS) in the cryoEM map (a) Modelling of $\mathrm{CHS} 1$ (green) in GIRK2 ${ }^{\mathrm{PIP} / \mathrm{CHS}}$ cryoEM map suggests poses with subtle differences in the orientation of iso-octyl tail. The density of CHS1 is contoured at a threshold level of 0.004 in ChimeraX. (b) Illustration of residues around CHS1 colored based on hydrophobicity (dark cyan - most hydrophilic, yellow - most hydrophobic) in ChimeraX, CHS1 is bound in a hydrophobic environment. (c) CHS2 (green) is also surrounded by hydrophobic residues including F186 (pink), V72, L79, I82, L86, L89, 197 and V101 (purple). The density of CHS2 is contoured at a threshold level of 0.0073 in ChimeraX. (d) Comparison of GIRK2 $2^{\mathrm{PIP} 2 / \mathrm{CHS}}$ (cyan, contoured at a threshold level of 0.0115 in ChimeraX) and GIRK2 $2^{\mathrm{PIP} 2 *}$ (violet, contoured at a threshold level of 0.0095 in ChimeraX) maps, the position of $\mathrm{CHS}$ (green) and $\mathrm{PIP}_{2}$ (red) molecules are as in GIRK2 ${ }^{\mathrm{PIP} / \mathrm{CHS}}$ structure. Densities observed in GIRK2 ${ }^{\mathrm{PIP} 2 / \mathrm{CHS}}$ map that were annotated as putative $\mathrm{CHS}$ sites are absent in GIRK2 ${ }^{\mathrm{PIP} 2 *}$ map. Other poorly resolved densities near $\mathrm{PIP}_{2}$ consistent in both maps (highlighted one of them in black box) might correspond to other putative $\mathrm{CHS}$ or phospholipid binding sites. 
a
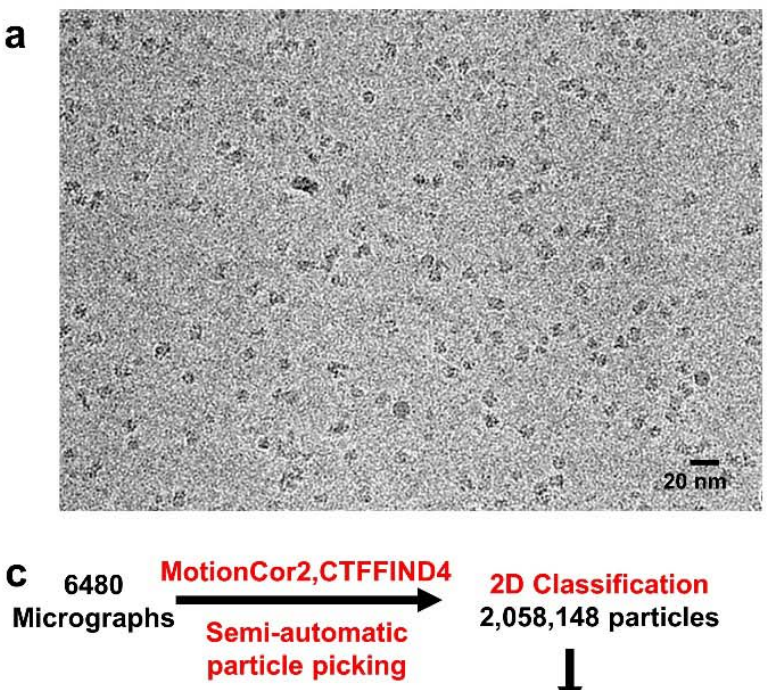

2D Classification 2,058,148 particles

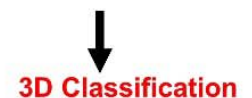
400,342 particles b
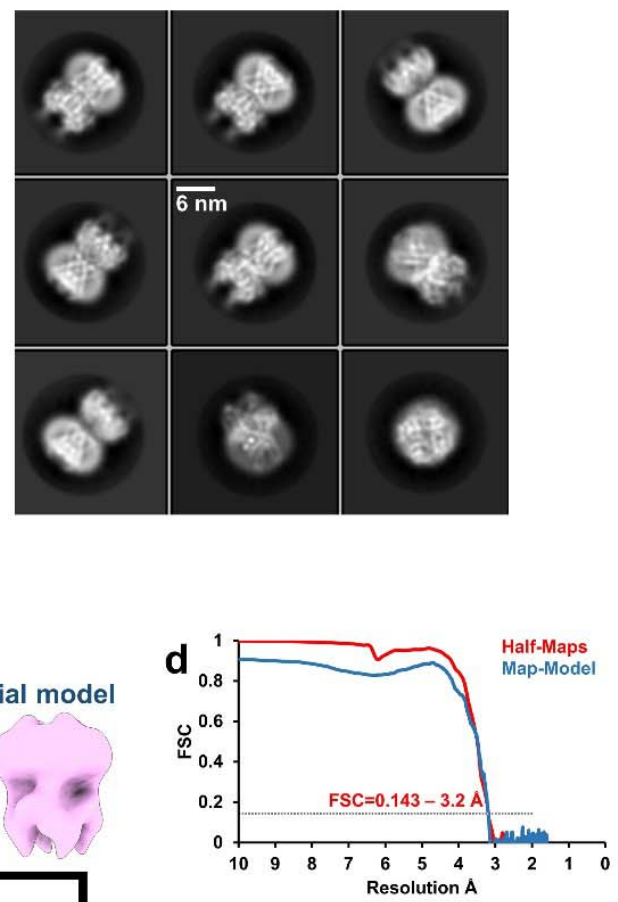

e

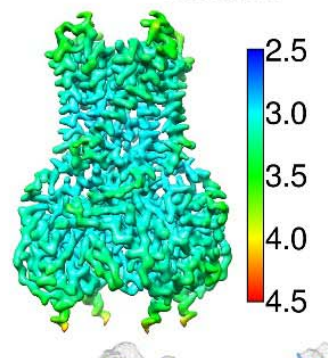

f

3D Refinement 154,071 particles

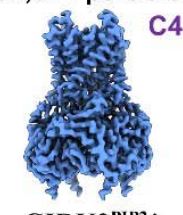

GIRK2 $^{\text {PIP2* } *}$ $3.2 \AA$

M2 helix h

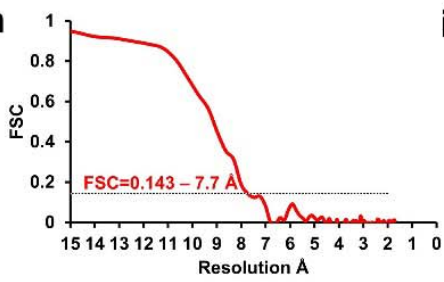

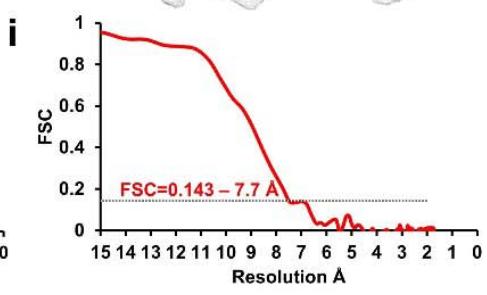

g

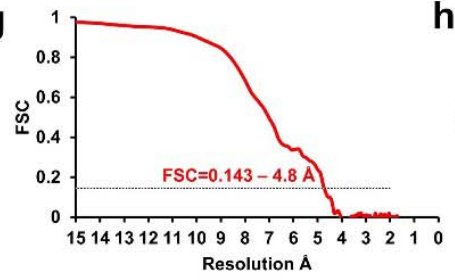

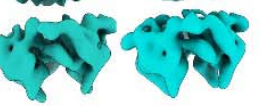

GIRK2 ${ }^{\text {PIP2 } * * *}$ GIRK2 $^{\text {PIP2 } * * * * *}$

$7.7 \AA$

Extended Figure S4: CryoEM data processing of GIRK2 in presence of PIP $_{2}$. (a) and (b) are representative cryoEM image and 2D averages. (c) Data processing flow chart. (d) FSC curve of $\mathrm{GIRK}^{\mathrm{PIP} 2 *}$, the dotted line indicating FSC at 0.143 . (e) Local resolution estimate of GIRK2 ${ }^{\mathrm{PIP} 2 *}$ by Relion. (f) Fit of the transmembrane helices M1 and M2 into the $3.2 \AA$ GIRK2 ${ }^{P I P 2 *}$ cryoEM map. (g) FSC curve of GIRK2 $2^{\mathrm{PIP} 2 \star *}$, the dotted line indicating FSC at 0.143 . (h) FSC curve of GIRK2 ${ }^{\mathrm{PIP} 2 \star \star *}$, the dotted line

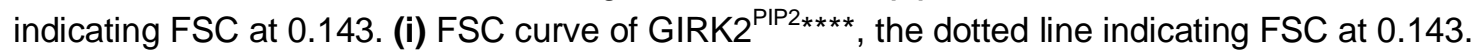



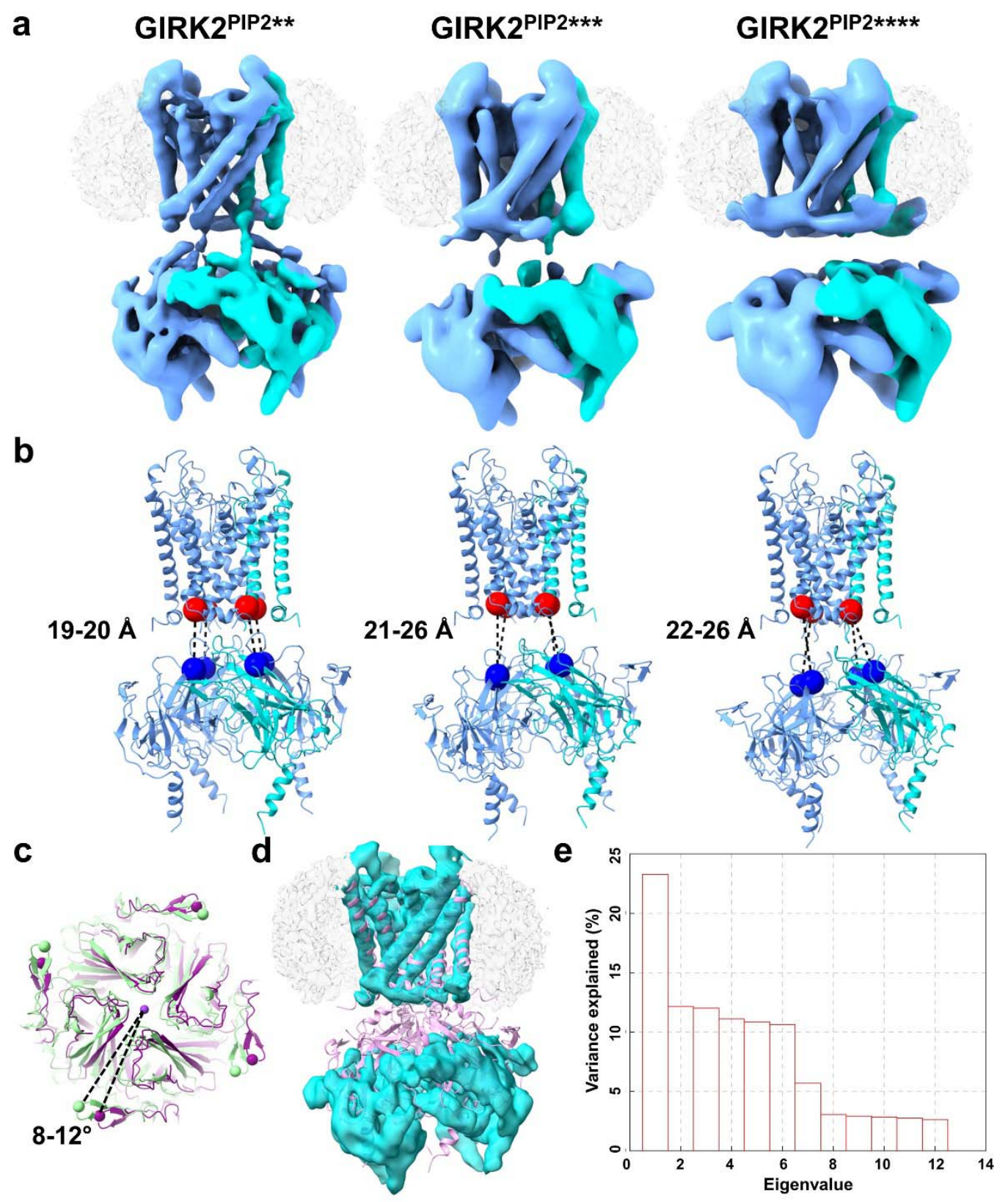

Extended Figure S5: Evaluation of CTD detachment and twist in different structures (a) CryoEM maps of GIRK2 $2^{\mathrm{PIP} 2 * \star}$, GIRK2 $2^{\mathrm{PIP} 2 * \star *}$ and GIRK2 $2^{\mathrm{PIP} 2 \star * \star *}$ with varying conformation of CTD with respect to TMD, one of the subunits is highlighted in cyan. (b) In these structures, CTD detachment is assessed by evaluating the distance between $\mathrm{Ca}$ atoms of residues I195 (denoted by red sphere) and Q322 (denoted by blue sphere). (c) Comparison of CTD twist of GIRK2 ${ }^{\mathrm{PIP} 2 * *}$ (purple) with $\mathrm{GIRK}^{\mathrm{PIP2} 2 *}$ (green) assessed by the angle of rotation of $\mathrm{Ca}$ atom of G347 (represented in sphere) around the four-fold axis after aligning the TMD near selectivity filter. (d) GIRK2 apo X-ray crystal structure (PDBID: 3SYO) is docked into GIRK2 apo cryoEM map. In contrast to the apo cryoEM map, the CTD in the crystal structure is engaged to the membrane. (e) Variance (\%) for different eigenvalues obtained by multibody refinement of GIRK2 apo with TMD and CTD as separate bodies. A movie depicting the movements between TMD and CTD in 3D models for principal component 1 is provided as Extended Movie S1. 

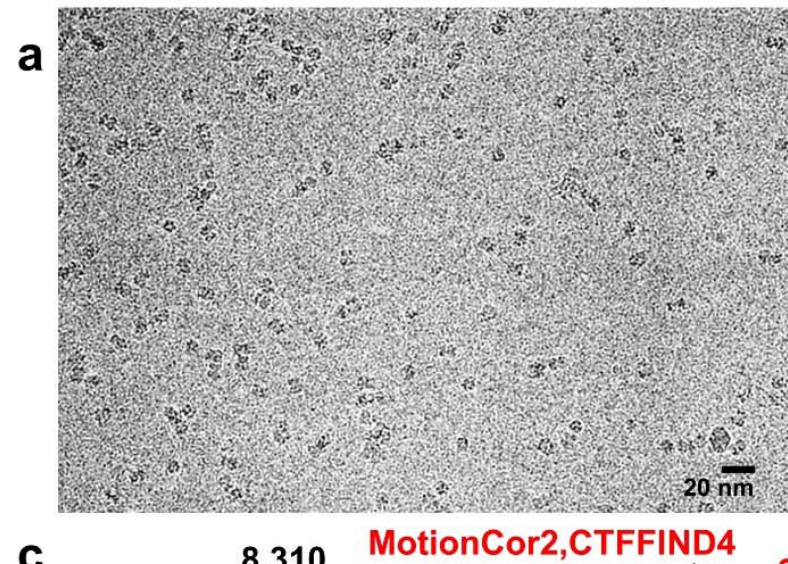

C

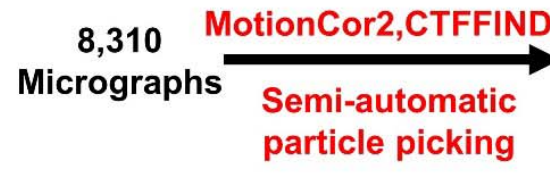

b

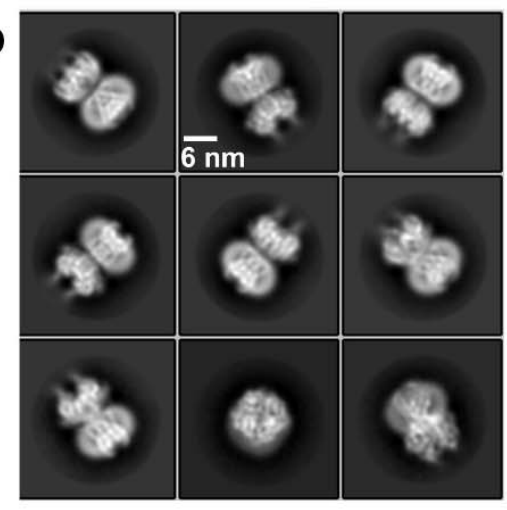

2D Classification

2,532,601 particles

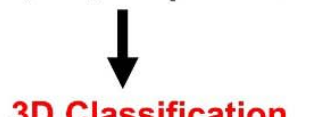

3D Classification

473,059 particles

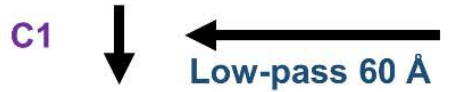

Initial model
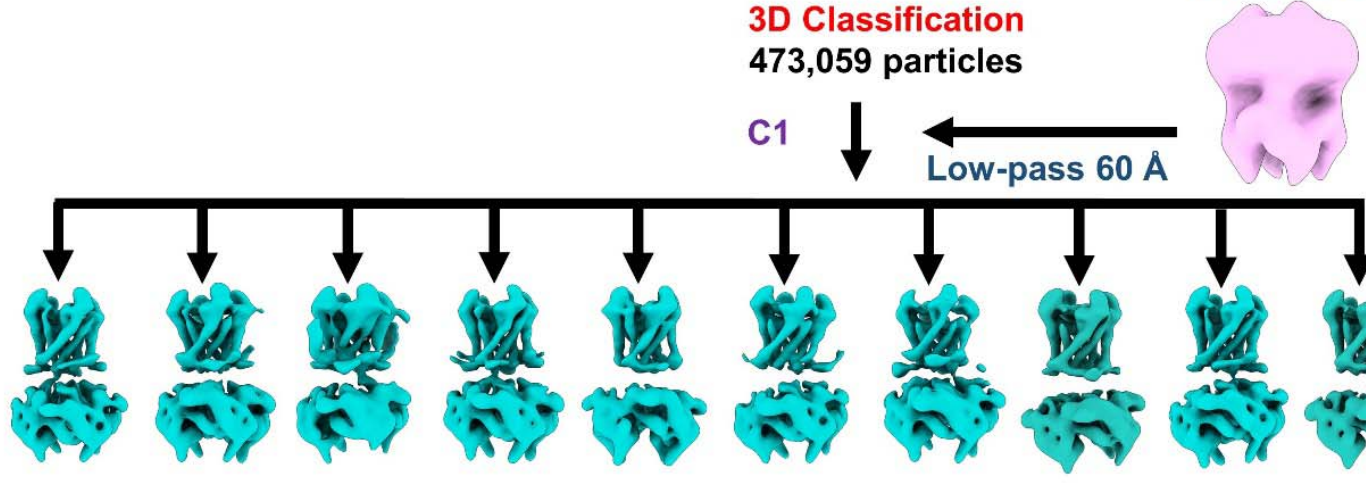

$13.6 \%$

$10.3 \%$

$4.6 \%$

$9.5 \%$

$8.2 \%$

$9.2 \%$

$9.3 \%$

d
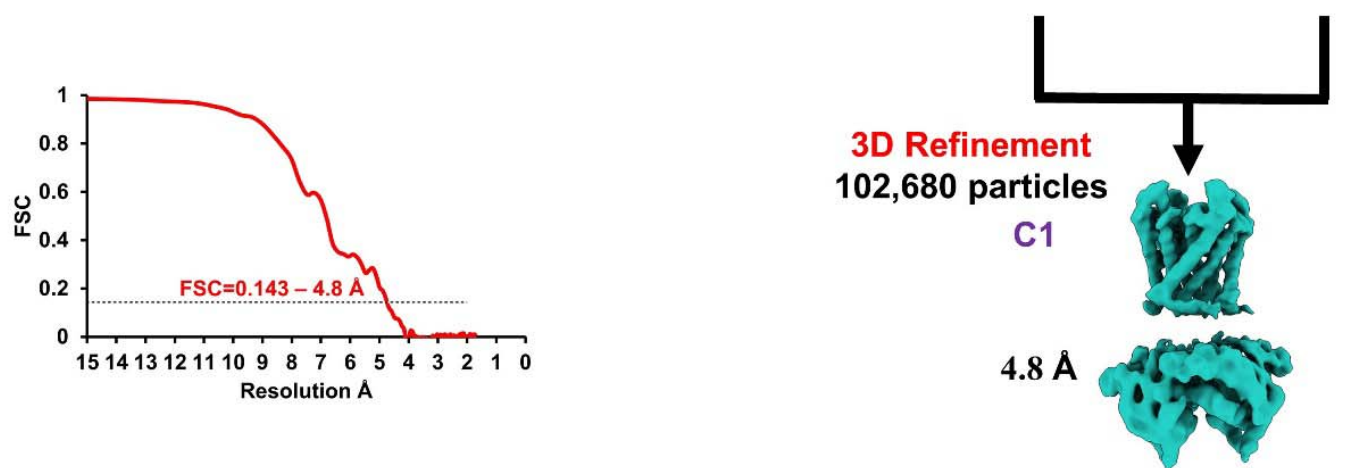

Extended Figure S6: CryoEM data processing of apo GIRK2. (a) and (b) are representative cryoEM image and 2D averages. (c) Data processing flow chart. (d) FSC curve, the dotted line indicating FSC at 0.143 . 

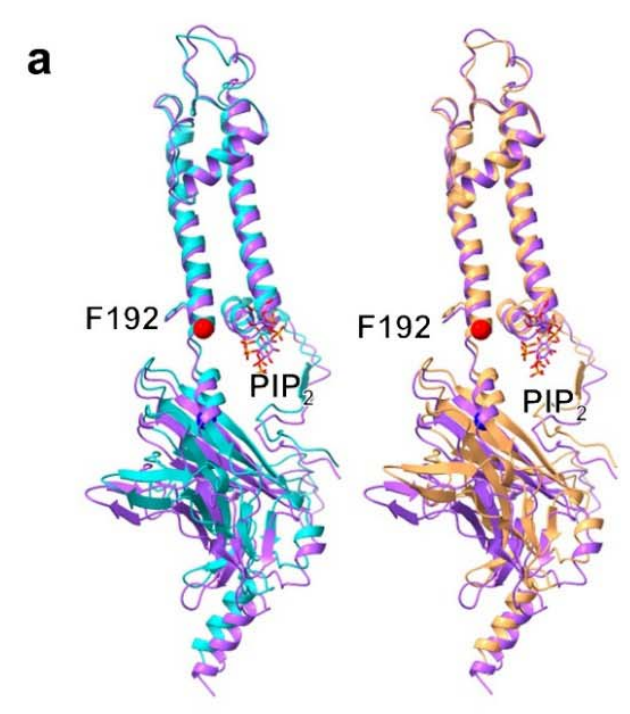

d
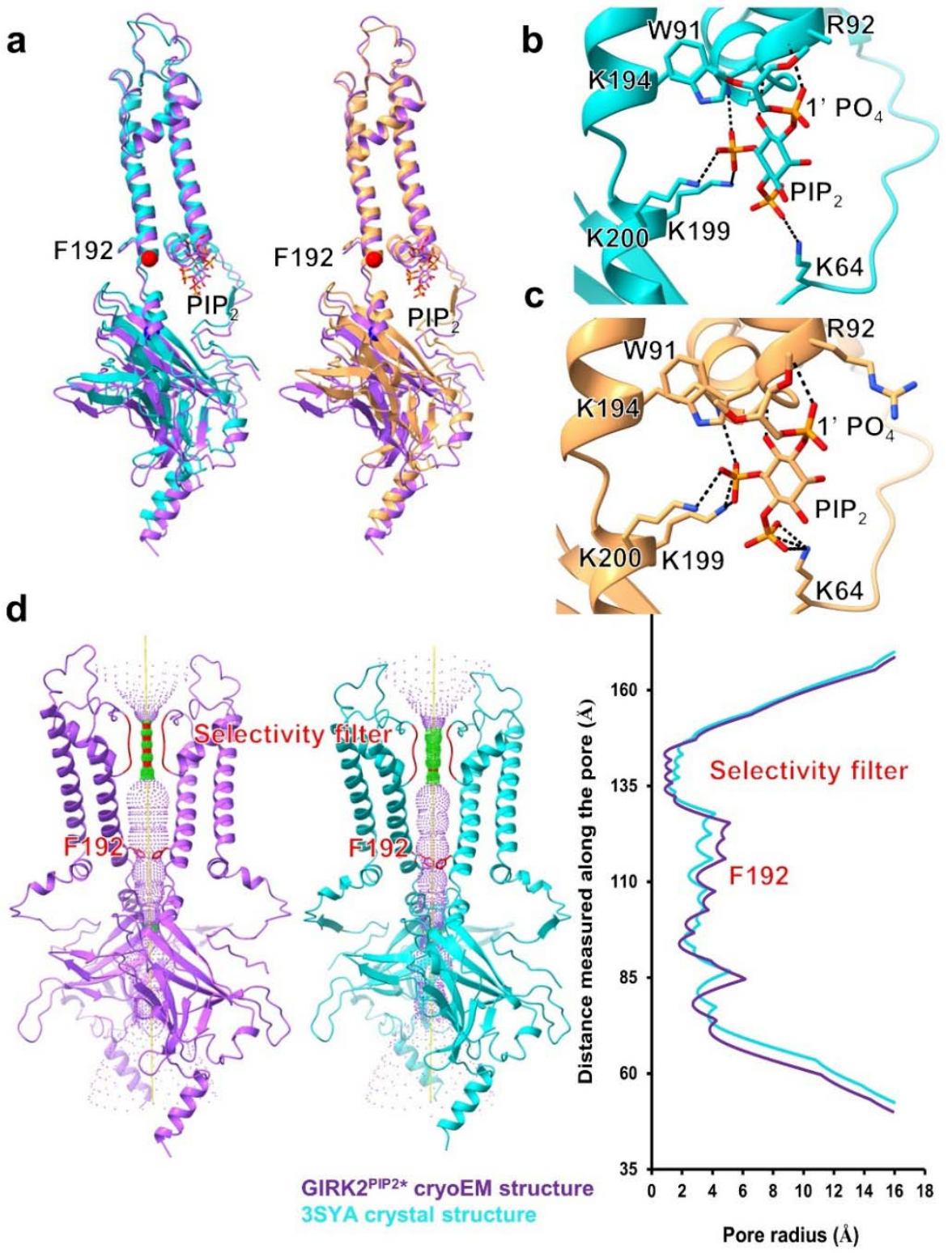

Extended Figure S7: Comparison of $\mathrm{PIP}_{2}$ coordination, CTD and pore in cryoEM and X-ray crystal structures. (a) Comparison of $\mathrm{PIP}_{2}$ binding pocket and CTD in GIRK2 ${ }^{\mathrm{PIP} 2 *}$ (violet), crystal structures with only $\mathrm{PIP}_{2}$ (PDBID:3SYA, blue) and with GßY and PIP 2 (PDBID:4KFM, orange) after aligning the TMD near selectivity filter. There are subtle differences in the position of $\mathrm{PIP}_{2}, \mathrm{CTD}$ and inner-helix gate residue F192. Compared to crystal structure with only $\mathrm{PIP}_{2}$ (3SYA), the positions of phosphate atom of $1{ }^{\prime} \mathrm{PO}_{4}$ of $\mathrm{PIP}_{2}$ and CTD (evaluated by distance between Ca atoms of 1195, red sphere and Q322, blue sphere) in GIRK2 ${ }^{\text {PIP2* }}$ cryoEM structure are moved away from the membrane towards the cytoplasmic side by $~ 3$ and $1 \AA$, respectively, and accompanied with $\sim 3^{\circ}$ rotation of CTD around the four-fold axis. (b) and (c) are $\mathrm{PIP}_{2}$ coordination in crystal structures PDBID:3SYA (blue) and PDBID:4KFM (orange), respectively. In crystal structures, $1^{\prime} \mathrm{PO}_{4}$ of $\mathrm{PIP}_{2}$ is coordinated by backbone amide of $\mathrm{R} 92$ in contrast to the side chain as seen in cryoEM structures. (d) Pore comparison using HOLE software for cryoEM structure GIRK2 ${ }^{\text {PIP2* }}$ (violet) and X-ray crystal structure PDBID:3SYA (blue). The pore radius around the inner-helix gate (F192) is wider in the cryoEM structure when compared to 3SYA. 
bioRxiv preprint doi: https://doi.org/10.1101/2020.06.04.134544; this version posted June 5, 2020. The copyright holder for this preprint (which was not certified by peer review) is the author/funder, who has granted bioRxiv a license to display the preprint in perpetuity. It is made available under aCC-BY-ND 4.0 International license.

Extended Movie S1: Movie depicting the models obtained from multibody refinement of GIRK2 apo with transmembrane (TMD) and cytoplasmic (CTD) domains as two separate bodies.

Filename: Extended Video S1_annot_small.MP4 


\begin{tabular}{|c|c|c|}
\hline & GIRK2 $2^{\mathrm{PIP} 2 / \mathrm{CHS}}$ & Apo \\
\hline Voltage (kV) & 300 & 300 \\
\hline Magnification & 58,824 & 58,824 \\
\hline Defocus $(\mu \mathrm{m})$ & -1.5 to -2.2 & -0.8 to -2.2 \\
\hline Pixel size (文) & 0.85 & 0.85 \\
\hline $\begin{array}{l}\text { Total electron dose } \\
\text { (electrons/pixel) }\end{array}$ & 60 & 60 \\
\hline Exposure time (s) & 3 & 3 \\
\hline No. of images & 642 & 8,310 \\
\hline No. of frames per image & 60 & 60 \\
\hline Initial particle number & 297,174 & $2,532,601$ \\
\hline $\begin{array}{l}\text { Particle number for 3D } \\
\text { classification }\end{array}$ & 84,912 & 473,059 \\
\hline Final particle number & 44,990 & 102,680 \\
\hline Resolution (Å) & 3.5 & 4.8 \\
\hline Symmetry imposed & $\mathrm{C} 4$ & $\mathrm{C} 1$ \\
\hline B-factor $\left(\AA^{2}\right)$ & 100 & 201 \\
\hline \multicolumn{3}{|c|}{ Model Refinement } \\
\hline Non-hydrogen atoms & 10,685 & \\
\hline \multicolumn{3}{|l|}{ R.M.S Deviations } \\
\hline Bond length $(\AA)$ & 0.004 & \\
\hline Bond angle $\left(^{\circ}\right)$ & 1.042 & \\
\hline \multicolumn{3}{|c|}{ Ramachandran plot } \\
\hline Favored (\%) & 91.7 & \\
\hline Allowed (\%) & 8.3 & \\
\hline Outlier (\%) & 0.0 & \\
\hline Clashscore & 13.7 & \\
\hline
\end{tabular}

Extended Table S1: Data collection and processing statistics of GIRK2 ${ }^{\mathrm{PIP} / \mathrm{CHS}}$ and apo structures. 


\begin{tabular}{|c|c|c|c|c|}
\hline & GIRK2 ${ }^{\mathrm{PIP} 2 *}$ & $\operatorname{GIRK} 2^{\mathrm{PIP2 \star \star}}$ & GIRK2 $^{\mathrm{PIP} 2 \star \star \star}$ & $\operatorname{GIRK} 2^{\mathrm{PIP} 2 \star \star \star \star}$ \\
\hline Voltage (kV) & 300 & 300 & 300 & 300 \\
\hline Magnification & 58,824 & 58,824 & 58,824 & 58,824 \\
\hline Defocus $(\mu \mathrm{m})$ & -0.8 to -2.2 & -0.8 to -2.2 & -0.8 to -2.2 & -0.8 to -2.2 \\
\hline Pixel size $(\AA ̊ \AA)$ & 0.85 & 0.85 & 0.85 & 0.85 \\
\hline $\begin{array}{l}\text { Total electron dose } \\
\text { (electrons/pixel) }\end{array}$ & 60 & 60 & 60 & 60 \\
\hline Exposure time (s) & 3 & 3 & 3 & 3 \\
\hline No. of images & 6480 & 6480 & 6480 & 6480 \\
\hline No. of frames per image & 60 & 60 & 60 & 60 \\
\hline Initial particle number & $2,058,148$ & $2,058,148$ & $2,058,148$ & $2,058,148$ \\
\hline $\begin{array}{l}\text { Particle number for 3D } \\
\text { classification }\end{array}$ & 400,342 & 400,342 & 400,342 & 400,342 \\
\hline Final particle number & 154,071 & 109,405 & 40,463 & 40,798 \\
\hline Resolution (A) & 3.2 & 4.8 & 7.7 & 7.7 \\
\hline Symmetry imposed & $\mathrm{C} 4$ & $\mathrm{C} 1$ & C1 & $\mathrm{C} 1$ \\
\hline B-factor $\left(\AA^{2}\right)$ & 60 & 100 & 60 & 60 \\
\hline \multicolumn{5}{|c|}{ Model Refinement } \\
\hline Non-hydrogen atoms & 10,544 & & & \\
\hline \multicolumn{5}{|l|}{ R.M.S Deviations } \\
\hline Bond length $(\AA)$ & 0.01 & & & \\
\hline Bond angle $\left(^{\circ}\right)$ & 1.16 & & & \\
\hline \multicolumn{5}{|c|}{ Ramachandran plot } \\
\hline Favored (\%) & 89.0 & & & \\
\hline Allowed (\%) & 11.0 & & & \\
\hline Outlier (\%) & 0.0 & & & \\
\hline Clashscore & 2.9 & & & \\
\hline
\end{tabular}

Extended Table S2: Data collection and processing statistics of GIRK2 ${ }^{\text {PIP2 }}$ structures. 
bioRxiv preprint doi: https://doi.org/10.1101/2020.06.04.134544; this version posted June 5, 2020. The copyright holder for this preprint (which was not certified by peer review) is the author/funder, who has granted bioRxiv a license to display the preprint in perpetuity. It is made available under aCC-BY-ND 4.0 International license.

\section{References}

1 Glaaser, I. W. \& Slesinger, P. A. Dual activation of neuronal G protein-gated inwardly rectifying potassium (GIRK) channels by cholesterol and alcohol. Sci Rep 7, 4592, doi:10.1038/s41598-017-04681-x (2017). 